\title{
APHIDS UNDER STRESS. SPECIES GROUPS AND ECOLOGICAL FUNCTIONAL GROUPS OF APHIDS DEFINE HEAVY METAL GRASSLANDS OF CENTRAL EUROPE
}

\author{
(*) Department of Entomology and Environmental Protection, Poznań University of Life Sciences, Dąbrowskiego 159, \\ 60-594 Poznań, Poland. \\ (**) The Upper Silesian Nature Society, Huberta 35, 40-543 Katowice, Poland \\ Corresponding author: e-mail: osiadacz@up.poznan.pl
}

Osiadacz B., Hałaj R. - Aphids under stress. Species groups and ecological functional groups of aphids define heavy metal grasslands of Central Europe.

Interest in trace metals and the environmental effects of their deposition significantly increased recently. Ecological communities formed on soils with a high concentration of heavy metals are characterised by a particular composition of plants and invertebrates in response to unfavourable physical and chemical conditions and under a strong selective pressure. Calaminarian grasslands as well as other dry grasslands are fragile habitats, very rare in Central Europe; such areas are often protected within nature reserves. This paper is the first comprehensive study of aphids (Insecta: Hemiptera: Aphidomorpha) of the metalliferous areas in Central Europe. It helped to describe the species diversity of aphid communities related to the plants of heavy metal grasslands, define the level of relationships between aphids and plants of heavy metal habitats and determine diagnostic aphid species for assemblages forming in such post-industrial landscapes. On the basis of ecological groups determined for aphids, also the number and percent of the species which form them and their ratios structure aphid communities and their condition was defined. An elevated heavy metal content in the soil does not limit the species richness of such an aphid community in comparison with those of other dry grasslands. However, these aphid communities possess specific features resulting from the mixture of species arriving from dry calcareous and sandy grasslands. The concept of ecological functional groups for analysis of aphid communities is introduced. Such an approach is useful for describing aphid communities in time and the directions of their changes, thus helping to monitor successive changes and the habitat state.

KEY WORDS: abiotic and biotic components, aphid community - plant community interactions, aphid diversity, semi-natural grasslands, unfriendly habitats.

\section{INTRODUCTION}

Heavy metal grasslands (also called calaminarian grasslands) are among the rarer plant communities in Europe. They form on soils naturally rich or artificially enriched in heavy metals (most frequently $\mathrm{Zn}, \mathrm{Pb}, \mathrm{Cd}$ ). In their typical form they occur in relatively few and isolated sites in southern and western Europe, particularly in Belgium, France, Germany and Italy (e.g. BECKER, 2012, EUROPEAN COMmission DG ENVIRONMENT, 2013). Small patches of this community, floristically slightly impoverished, also exist in Central Europe - near the southern Polish town of Olkusz on the Silesia-Kraków Upland - in areas with abundant deposits of zinc and lead ores. The mining of these deposits since the Middle Ages has significantly changed the local landscape, leaving deep excavations and mining waste landfills. In such an unfriendly habitat (shallow, skeletal, dry, nutrient-poor soils with a high content of heavy metals), a long succession spontaneously formed a typical combination which contains calaminarian grassland species (e.g. GRODZIŃSKA and SZAREKŁuKASZEWSKA, 2009, SZAREK-ŁUKASZEWSKA and GRODZIŃSKA, 2011). Similar plants can also be found in landfill sites of waste from heavy metal works in various parts of Poland, but particularly in Upper Silesia (e.g. ROSTAŃSKI, 1997a, MAŃCZYK and ROSTAŃSKI, 2003).

Owing to the unique character of the communities formed on soils with excess heavy metal contents and their rarity in Europe, the dry grassland communities of
Violetalia calaminariae Br.-B1. et R.Tx. 1943 have been included in the European Natura 2000 network of special areas of conservation (code 6130). Protection under the EU Habitats Directive $e^{1}$ in Poland covers three such areas, two of which are in the Olkusz area: 'Armeria' (area code PLH120091) and 'Pleszczotka' (PLH120092).

Ecological communities (biocoenoses) formed on soils with high levels of heavy metals have a unique flora and fauna (BAKER et al., 2010). The extremely unfavorable physical and chemical conditions (abiotic components) of such habitats (high soil toxicity, high temperature, shortage of nutrients and water) exert a strong selection pressure on biotic components. In response to this pressure and the ensuing rapid microevolutionary processes, new ecotypes or kinds of plants appear; they then form (by matching) communities of high resistance, which makes them more durable (WIERZBICKA and RosTAŃSKI, 2002). Studies of plants, i.e. the producer level in a food chain, which grow in metalliferous areas have been conducted for several decades (DOBRZAŃSKA, 1955) and are still a focus of interest (NowAK et al., 2011). On the other hand, the wildlife of such habitats is poorly known. It would be interesting to find out whether the pressure exerted on plants could also affect a higher level of the food pyramid, i.e. primary

\footnotetext{
' Council Directive 92/43/EEC of 21 May 1992 on the conservation of natural habitats and of wild fauna and flora [OJ L 206, 22.7.1992, p.7]
} 
consumers, particularly insects. Sucking phytophages, such as Sternorrhyncha, especially aphids (Aphidomorpha), are models for this type of study. Most of them are monophagous or narrowly oligophagous and are characterized by a very close relationship with their host plant (EASTOP, 1995; LiN et al., 2015; OsIADACZ and HAŁAJ, 2009). Such types of feeding are among the significant factors governing the possibility of specific aphid communities (aphidocoenoses) being formed in specific plant communities. This has been corroborated by the studies of many authors performed in various habitats (DURAK and WOJCIECHOWSKI, 2005; OsIADACZ and WoJCIECHOWSKI, 2008; DEPA and WoJCIECHOWSKI, 2009), including those with extreme living conditions (SzElEGIEWICZ, 1974; CzYLOK and WoJCIECHOWSKI, 1987; WOJCIECHOWSKI et al., 1989). Despite the numerous papers describing aphid communities, only one of them focuses on aphids in metalliferous areas (CZYLOK et al., 1991).

We therefore decided to address this topic and attempt to answer basic questions

Which aphid species occur in metalliferous areas, how numerous are they and which habitats are they found in?

Can specific aphid communities form in such characteristic habitats? If so, what is the species structure of their aphid groups? What can make them different from the aphid communities formed in habitats with similar physical and chemical soil parameters (particularly temperature and humidity), but without an excess of heavy metals?

\section{MATERIALS AND METHODS}

\section{SITE DESCRIPTION}

The study covered 5 sites (plots) located in southern Poland in the Silesia-Kraków Upland (Fig. I). Two of the sites, 'Pleszczotka' (P-1) and 'Armeria' (P-2), are characterized by heavy metal grasslands (of Violetea calaminariae class) (SZAREK-ŁUKASZEWSKA and GRODZIŃSKA, 2011; KAPUSTA et al., 2010; KowOLIK et al., 2010). The third site, 'Zgoda' (P-3), is an over 80-year-old metallurgical dump with similar types of plant communities that tolerate high concentrations of heavy metals in the soil (ROSTAŃSKI, 1997b; SKuBAŁA, 2011). These communities correspond to the last stages of plant succession on such dumps, i.e. the formation of a grass sward and overgrowth by young trees (GRESZTA and MORAWSKI, 1972).

Two control plots were selected for comparative analyses with the material collected from the above-mentioned sites. They had similar microclimatic conditions (temperature, humidity), but not the extreme pressure of human factors, i.e. unnaturally high levels of toxic heavy metals in the ground. The first site - 'Równa Góra' (CP-1) (near Strzyżowice) was representative of dry calcareous grassland [of FestucoBrometea Br. Bl. et R.Tx. 1943 class (BABCZYŃSKA-SENDEK, 2005)], while the other one - 'Rabsztyn' (CP-2) (Rabsztyn near Olkusz), represented sandy grassland [of Koelerio glaucae-Corynephoretea canescentis Klika in Klika et Novak 1941 class (MATUSZKIEWICZ, 2012)].

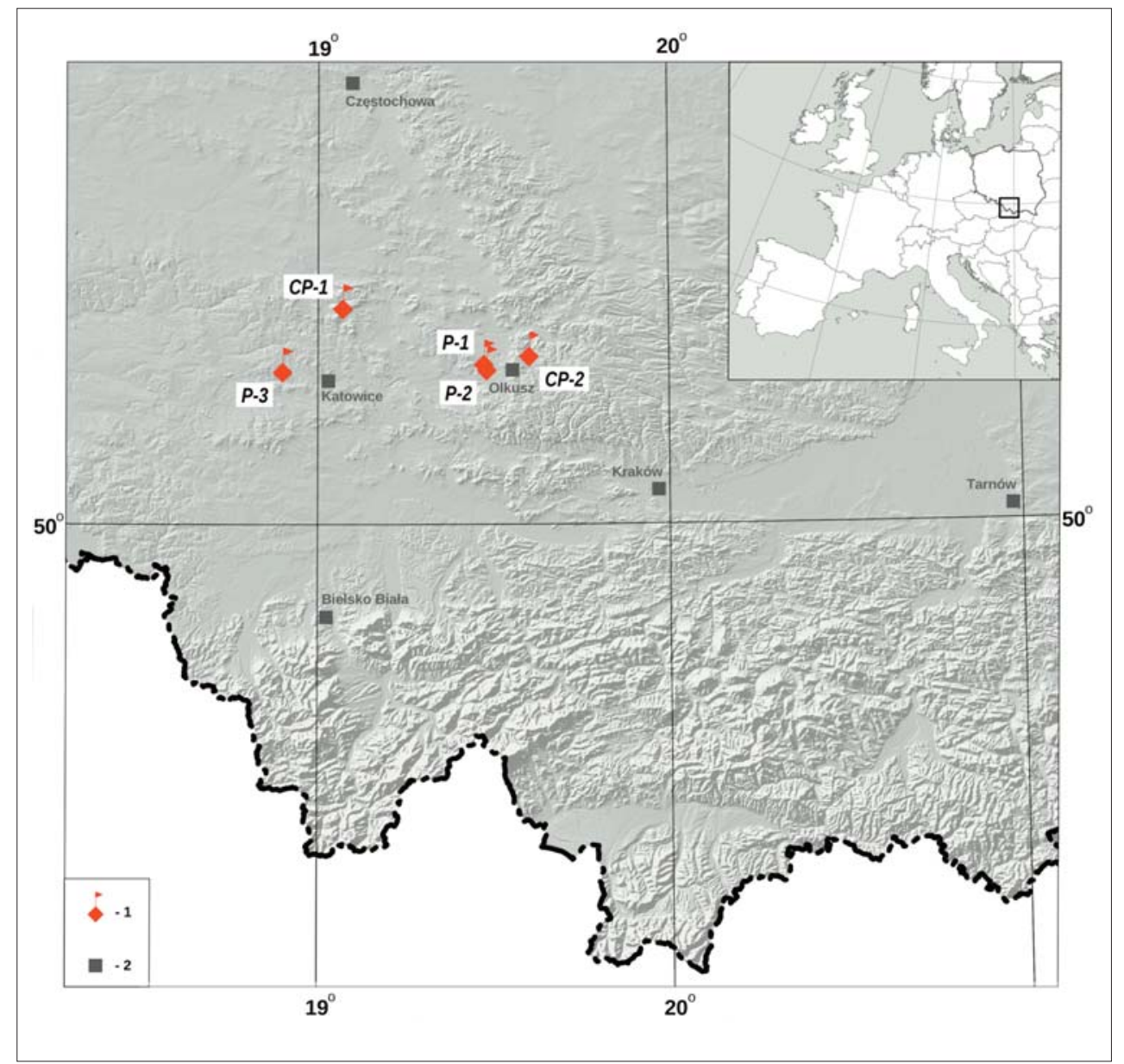

Fig. I - Study area with location of the sampling plots (1): P-1 'Pleszczotka', P-2 'Armeria', P-3 'Zgoda', CP-1 'Równa Góra', CP-2 'Rabsztyn'; 2 - the main cities. 
In terms of phytosociology all the 5 sites are dry grasslands in a wide sense. They represent similar geobotanical conditions, with similar plant coverage, plant-species composition and the same floristic diversity (which means the number of plant species per area unit).

\section{MATERIAL COLLECTION AND DATA ANALYSIS}

We carried out the studies from June to the end of July 2011-2013 by visiting the study plots at two-week intervals. All the plots were sampled in the same way, with the same methods and intensity. The same selected plots of $100 \mathrm{~m}^{2}$ were explored each time.

The material was obtained using the standard methods of aphid collection and preparation (QUEDNAU, 2003; BLACKMAN and EASTOP, 2015; HalaJ and OsIADACZ, 2016). These comprised collecting the aphids by hand following visual inspection, brushing aphids from host plants to white sheet and using the sweep net. The aphids were classified with collective and, if necessary, specialist keys, an exhaustive list of which is presented in the paper by BLACKMAN and EASTOP (2015). The evidence material in the form of microscopic slides is deposited in Department of Entomology and Environmental Protection of Poznan University of Life Sciences.

\section{PLANT SPECIES WERE DETERMINED AT STUDY PLOTS.}

Based on the paper by JĘDRZEJCZYK-KORYCIŃSKA (2011) we divided the host plant species into three groups: plants displaying a very high, high and moderate degree of attachment to metalliferous areas. We also indicated the aphid species found there along with the degree of their feeding type as: monophagous, oligophagous and polyphagous species (Table 1).

Taking into consideration the information from Table 1 we analysed the level of plant relation to metalliferous areas and the level of aphid relation to these plants (feeding type). Based on it and the papers which applied the 'fidelity class' concept concerning communities of aphids and other Hemiptera (e.g. GoRCZYCA, 1994; OsiadACZ and HAŁAJ, 2015; SZWEDO et al., 1998), we defined the fidelity of aphid species to the plant communities formed in the study plots by adopting the following criteria:

- differential species - aphids (monophagous or narrowly oligophagous) trophically related to plant species with a very close connection to calaminarian grasslands and occurring only in metalliferous areas;

- characteristic species - aphids (monophagous or narrowly oligophagous) trophically related to plant species which tolerate a higher content of heavy metals in the soil, but occurring not only in metalliferous areas;

- accompanying species - aphids occurring in metalliferous areas but not only trophically connected with plant species tolerating a higher content of heavy metals in the soil (broadly oligophagous), or monophagous and narrowly oligophagous not connected with plants tolerating a higher content of heavy metals in the soil and also occurring in metalliferous areas;

- indifferent species - oligophagous and polyphagous with no clear predisposition for any plant community.

We analyzed also the aphid species structure in the study areas. We took into consideration the entire set of correlations between particular aphid species and their host plants: feeding (trophic criterion), changes of host plant during the life cycle and type of reproduction (the occurrence or lack of a sexual generation), the type of growth of the host plant (life form in the sense of Raunkiaer system according to position of buds during winter), the range centre of plant species in particular community types and its 'diagnostic ability' for such communities (according to studies by phytosociologists, e.g. BRAUN-BlanQueT, 1964; DeNGLER et al., 2012; MATUSZKIEWICZ, 2012). Considering these criteria, we divided aphid species into ecological functional groups (EFG) (two groups within each of which we defined two subgroups):

- 'S' (specialists) - monophagous and narrowly oligophagous, mainly monoecious and holocyclic aphid species:

- 'Fr-S' (specialists in forest zones) - related to tree-andshrub plant formations,

- 'NFr-S' (specialists in non-forest zones) - related to forest-free formations, dense grass-herbaceous;

- 'G' (generalists, opportunists) - polyphagous and oligophagous, mainly heteroecious (rarely monoecious) holocyclic or anholocyclic aphid species:

- 'Fr-G' (generalists in forest zones) - related to treeand-shrub communities,

- 'NFr-G' (generalists in non-forest zones) - related to forest-free grass-herbaceous communities.

Based on the above assumptions we analysed the classification of aphids into particular 'EFG' and presented its quantitative and percentage results in a graphic form (Fig. II).

On their basis, in order to characterise the structure of interesting aphid communities in more detail, we calculated the proportions between ' $\mathrm{EFG}$ ' groups $\left(\mathrm{RP}_{\mathrm{EFG}}\right.$ - ratio of participations of ecological functional groups), according to a simple formula:

$$
\mathbf{R P}_{\mathrm{EFG}}=\boldsymbol{\alpha}_{\mathrm{EFG}} / \boldsymbol{\beta}_{\mathrm{EFG}} ;
$$

where: $\alpha_{\mathrm{EFG}}-$ number or percentage (share) of aphid species of one particular 'EFG', $\beta_{\mathrm{EFG}}$ - number or percentage (share) of aphid species of another particular 'EFG' (Table 2).

In order to compare the species composition of aphids (aphids communities) occurring on particular plots we used multivariate statistics: cluster analysis with Euclidean distance and two clustering method i.e. UPGMA (Average) and Ward method (EvERITT et al., 2011) (Fig. III).

To examine relationship between disturbance degree of particular plots and the ratios of distinguished ecological functional groups $\left(\mathrm{RP}_{\mathrm{EFG}}\right)$ simple linear regression was applied (Fig. IV). Ranks from 1 to 5 were assigned to particular study sites. Prior the analyses normality of distribution of response variable was checked by ShapiroWilk test (Table 2).

All statistics were conducted using $\mathrm{R}$ language and environment (R Core Team 2014).

\section{RESULTS}

Our studies yielded a total of 125 aphid species (Table 3 ). We recorded 102 aphid species $(81.6 \%$ of the total material) on the sites with heavy metal plants ('P' - plots P-1, P-2, P3 together), 76 species $(60.8 \%)$ on the control plots (' $\mathrm{CP}$ ' CP-1 \& CP-2), and found 51 species (40.8\%) common to both, i.e. ones which occurred in both metalliferous areas and control plots. On the other hand, we recorded 49 species $(39.2 \%)$ only from the sites with heavy metal plants, and $25(20 \%)$ only from the control plots (Fig. V). Overall, we found a higher number of aphid species on particular sites of the metalliferous areas (the most $(69 ; 55.2 \%)$ in the post-industrial dump, P-3) than on the control plots; we found the fewest $(45 ; 36 \%)$ in dry calcareous grasslands, CP-1 (Table 3, Fig. V.). 
Table 1 - Aphids found on heavy metal plants: I - plants with a very high degree of attachment to metalliferous areas, II - plants with a high degree of attachment for metalliferous areas, III - plants with moderate degree of attachment to metalliferous areas, A - anholocyclic, $\mathrm{HO}$ - holocyclic, $\mathrm{M}$ - monoecious, $\mathrm{H}$ - heteroecious, MN - monophagous, NOL - narrow oligophagous, OL - oligophagous, $\mathrm{P}$ polyphagous, 1 - indifferent species, 2 - accompanying species, 3 - characteristic species, 4 - differential species, $(*)$ - only for local of aphid communities, $(* *)$ - for all study of aphid communities.

\begin{tabular}{|c|c|c|c|c|c|c|c|c|}
\hline & \multirow{3}{*}{ Host plants } & \multicolumn{5}{|c|}{ Aphids in: } & \multirow{3}{*}{ Phagism } & \multirow{3}{*}{$\begin{array}{l}\text { Fidelity } \\
\text { class }\end{array}$} \\
\hline & & \multicolumn{3}{|c|}{ Calaminarian grasslands } & \multirow{2}{*}{$\begin{array}{c}\begin{array}{c}\text { Dry calcareous } \\
\text { grasslands }\end{array} \\
\text { CP-1 }\end{array}$} & \multirow{2}{*}{$\begin{array}{c}\begin{array}{c}\text { Sandy } \\
\text { grasslands }\end{array} \\
\text { CP-2 }\end{array}$} & & \\
\hline & & P-1 & P-2 & P-3 & & & & \\
\hline & $\begin{array}{l}\text { Cardaminopsis } \\
\text { arenosa }\end{array}$ & L. erysimi & L. erysimi & L. erysimi & - & L. erysimi & $\mathrm{OL}$ & 2 \\
\hline & Daucus carota & $\begin{array}{l}\text { A. lambersi } \\
\text { A. fabae }\end{array}$ & A. lambersi & A. lambersi & A. lambersi & A. lambersi & $\begin{array}{c}\text { NOL } \\
\mathrm{P}\end{array}$ & $\begin{array}{c}3(* *) \\
1\end{array}$ \\
\hline & $\begin{array}{l}\text { Euphorbia } \\
\text { cyparissias }\end{array}$ & A. euphorbiae & A. euphorbiae & - & A. euphorbiae & $\begin{array}{l}\text { A. euphorbiae } \\
\text { M. euphorbiae }\end{array}$ & $\begin{array}{c}\text { NOL } \\
\mathrm{P}\end{array}$ & $\begin{array}{c}3(*) \\
1\end{array}$ \\
\hline & Festuca ovina & $\begin{array}{l}\text { A. corni } \\
\text { F. formicaria }\end{array}$ & $\begin{array}{l}\text { A. corni } \\
\text { F. formicaria }\end{array}$ & F. formicaria & F. formicaria & - & $\begin{array}{l}\mathrm{P} \\
\mathrm{P}\end{array}$ & $\begin{array}{l}1 \\
1\end{array}$ \\
\hline \multirow[t]{7}{*}{$\mathbf{I}$} & $\begin{array}{l}\text { Leontodon } \\
\text { hispidus }\end{array}$ & $\begin{array}{l}\text { U. leontodontis } \\
\text { U. hypochoeridis }\end{array}$ & U. leontodontis & $\begin{array}{l}\text { A. leontodontis } \\
U . \text { leontodontis } \\
\text { U. hypochoeridis }\end{array}$ & U. leontodontis & U. leontodontis & $\begin{array}{l}\text { NOL } \\
\text { NOL } \\
\text { OL }\end{array}$ & $\begin{array}{c}3(*) \\
3(* *) \\
2\end{array}$ \\
\hline & $\begin{array}{l}\text { Lotus } \\
\text { corniculatus }\end{array}$ & $\begin{array}{l}\text { A. craccivora } \\
\text { A. loti } \\
\text { T. trifolii trifolii }\end{array}$ & $\begin{array}{l}\text { A. craccivora } \\
\text { A. loti } \\
\text { T. trifolii trifolii }\end{array}$ & $\begin{array}{l}\text { A. loti } \\
\text { T. trifolii trifolii }\end{array}$ & $\begin{array}{l}\text { A. craccivora } \\
- \\
\text { T. trifolii trifolii }\end{array}$ & $\begin{array}{l}- \\
- \\
-\end{array}$ & $\begin{array}{l}\mathrm{P} \\
\mathrm{OL} \\
\mathrm{OL}\end{array}$ & $\begin{array}{l}1 \\
2 \\
2\end{array}$ \\
\hline & $\begin{array}{l}\text { Pimpinella } \\
\text { saxifraga }\end{array}$ & A. subnitida & A. subnitida & - & A. subnitida & - & NOL & $3(*)$ \\
\hline & $\begin{array}{l}\text { Plantago } \\
\text { lanceolata }\end{array}$ & D. aucupariae & $\begin{array}{l}\text { B. lucifugus } \\
\text { D. aucupariae }\end{array}$ & D. aucupariae & - & B. lucifugus & $\begin{array}{l}\mathrm{MN} \\
\mathrm{NOL}\end{array}$ & $\begin{array}{c}3(*) \\
2\end{array}$ \\
\hline & $\begin{array}{l}\text { Ranunculus } \\
\text { acris }\end{array}$ & A. fabae & - & - & - & - & $\mathrm{P}$ & 1 \\
\hline & Silene vulgaris & B. populi & B. populi & B. populi & B. populi & B. populi & NOL & $3(* *)$ \\
\hline & $\begin{array}{l}\text { Achillea } \\
\text { millefolium }\end{array}$ & $\begin{array}{l}\text { M. millefolii } \\
\text { T. vandergooti }\end{array}$ & $\begin{array}{l}\text { M. millefolii } \\
\text { T. vandergooti }\end{array}$ & $\begin{array}{l}\text { M. millefolii } \\
\text { T. vandergooti }\end{array}$ & $\begin{array}{l}\text { M. millefolii } \\
\text { T. vandergooti }\end{array}$ & T. vandergooti & $\begin{array}{l}\mathrm{OL} \\
\mathrm{OL}\end{array}$ & $\begin{array}{l}2 \\
2\end{array}$ \\
\hline \multirow[t]{8}{*}{ II } & Pinus sylvestris & $\begin{array}{l}\text { C. pinea } \\
- \\
\text { C. pini } \\
\text { E. agilis } \\
\text { P. pini } \\
\text { C. }(S) \text {. pineti }\end{array}$ & $\begin{array}{l}\text { C. pinea } \\
\text { C. pilosa } \\
\text { C. pini } \\
- \\
- \\
\text { C. }(S) \text {. pineti }\end{array}$ & $\begin{array}{l}\text { C. pinea } \\
- \\
- \\
\text { E. agilis } \\
\text { P.pini } \\
\text { C. }(S) \text {. pineti }\end{array}$ & $\begin{array}{l}\text { C. pinea } \\
- \\
- \\
- \\
- \\
\text { C. (S). pineti }\end{array}$ & $\begin{array}{l}\text { C. pinea } \\
- \\
\text { C. pini } \\
\text { E. agilis } \\
- \\
\text { C. (S). pineti }\end{array}$ & $\begin{array}{l}\text { NOL } \\
\text { NOL } \\
\text { NOL } \\
\text { NOL } \\
\text { NOL } \\
\text { NOL }\end{array}$ & $\begin{array}{l}2 \\
2 \\
2 \\
2 \\
2 \\
2\end{array}$ \\
\hline & Rumex acetosa & A. rumicis & A. rumicis & A. rumicis & - & - & $\mathrm{OL}$ & 2 \\
\hline & \begin{tabular}{|l} 
Scabiosa \\
ochroleuca
\end{tabular} & $\begin{array}{l}\text { A. thomasi } \\
\text { M. rosae }\end{array}$ & $\begin{array}{l}\text { A. thomasi } \\
\text { M. rosae }\end{array}$ & - & - & - & $\begin{array}{c}\mathrm{OL} \\
\mathrm{P}\end{array}$ & $\begin{array}{l}2 \\
1 \\
\end{array}$ \\
\hline & $\begin{array}{l}\text { Thymus } \\
\text { pulegioides }\end{array}$ & A. serpylli & A. serpylli & - & A. serpylli & - & $\mathrm{OL}$ & 2 \\
\hline & $\begin{array}{l}\text { Anthyllis } \\
\text { vulneraria }\end{array}$ & A. klimeschi & - & - & A. klimeschi & - & $\mathrm{MN}$ & $3(*)$ \\
\hline & $\begin{array}{l}\text { Arrhenatherum } \\
\text { elatius }\end{array}$ & $\begin{array}{l}\text { S. maydis } \\
\text { S. avenae }\end{array}$ & S. maydis & - & S. avenae & - & $\begin{array}{l}\mathrm{P} \\
\mathrm{P}\end{array}$ & $\begin{array}{l}1 \\
1\end{array}$ \\
\hline & $\begin{array}{l}\text { Centaurea } \\
\text { scabiosa }\end{array}$ & A. centaureae & U. jaceae & U. jaceae & $\begin{array}{l}\text { A. centaureae } \\
\text { U. jaceae }\end{array}$ & - & $\begin{array}{l}\text { NOL } \\
\text { OL }\end{array}$ & $\begin{array}{c}3(*) \\
2\end{array}$ \\
\hline & Coronilla varia & A. pisum pisum & A. pisum pisum & - & A. pisum pisum & - & $\mathrm{P}$ & 1 \\
\hline \multirow[t]{5}{*}{ III } & $\begin{array}{l}\text { Euphrasia } \\
\text { stricta }\end{array}$ & - & - & B. pistaciae & - & - & $\mathrm{P}$ & 1 \\
\hline & Galium mollugo & $\begin{array}{l}\text { A. galiiscabri } \\
\text { A. molluginis }\end{array}$ & A. galiiscabri & A. galiiscabri & A. galiiscabri & - & $\begin{array}{l}\text { NOL } \\
\text { NOL }\end{array}$ & $\begin{array}{l}3(*) \\
3(*)\end{array}$ \\
\hline & $\begin{array}{l}\text { Helianthemum } \\
\text { nummularium }\end{array}$ & A. helianthemi & A. helianthemi & - & A. helianthemi & - & NOL & $3(*)$ \\
\hline & $\begin{array}{l}\text { Hieracium } \\
\text { pilosella }\end{array}$ & $\begin{array}{l}\text { A. pilosellae } \\
- \\
-\end{array}$ & $\begin{array}{l}\text { A. pilosellae } \\
- \\
-\end{array}$ & $\begin{array}{l}- \\
- \\
-\end{array}$ & $\begin{array}{l}- \\
- \\
-\end{array}$ & $\begin{array}{l}\text { A. pilosellae } \\
\text { N. pilosellae } \\
\text { U. obscurum }\end{array}$ & $\begin{array}{l}\text { NOL } \\
\text { NOL } \\
\text { NOL }\end{array}$ & $\begin{array}{l}3(*) \\
3(*) \\
3(*)\end{array}$ \\
\hline & $\begin{array}{l}\text { Rumex } \\
\text { thyrsiflorus }\end{array}$ & A. rumicis & A. rumicis & A. rumicis & - & - & $\mathrm{OL}$ & 2 \\
\hline
\end{tabular}




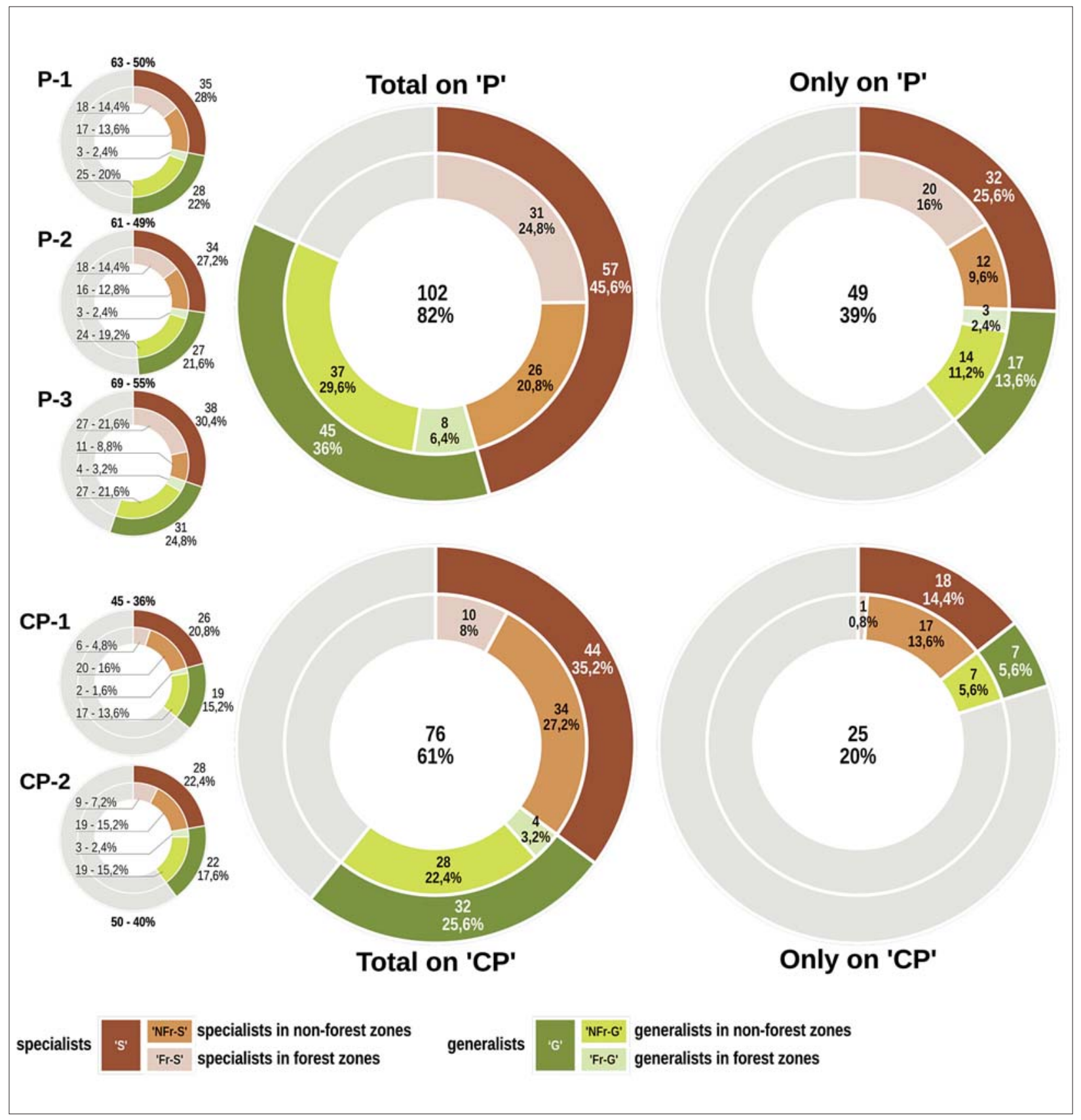

Fig. II - The number of aphid species of particular ecological functional groups (EFG) in the aphid communities of examined plots: 'Total on ... ' - the number of all the species of a particular EFG, 'Only on...' - the number of species of a particular EFG, which occurred only on 'P' or only on 'CP'.

Table 2 - The ratio of participations $\left(\mathrm{RP}_{\mathrm{EFG}}\right)$ in the aphid communities on examined plots and results of linear regression $\left(R^{2}, F, P\right)$ : ' $\mathrm{G}$ ' - generalists, 'Fr-G' - generalists in forest zones, 'NFr-G' - generalists in non-forest zones, 'S' - specialists, 'Fr-S' - specialists in forest zones, 'NFr-S' - specialists in non-forest zones, bolded values are significant at $P<0.05$.

\begin{tabular}{|l|c|c|c|c|c|c|c|r|}
\hline \hline \multirow{2}{*}{ Variable } & & & $\mathrm{RP}_{\mathrm{EFG}}$ & & & \multirow{2}{*}{$R^{2}$} & \multirow{2}{*}{$F$} & \multirow{2}{*}{$P$} \\
\cline { 2 - 7 } & $\mathrm{CP}-1$ & $\mathrm{CP}-2$ & $\mathrm{P}-1$ & $\mathrm{P}-2$ & $\mathrm{P}-3$ & & & \\
\hline 'S'/ 'G' & 1,37 & 1,27 & 1,25 & 1,26 & 1,22 & $\mathbf{0 , 7 9}$ & 11,46 & 0,042 \\
\hline 'Fr-S' / 'Fr-G' & 3 & 3 & 6 & 6 & 6,75 & $\mathbf{0 , 8 3}$ & 15,06 & 0,0303 \\
\hline 'NFr-S' / 'NFr-G' & 1,18 & 1 & 0,68 & 0,66 & 0,41 & $\mathbf{0 , 9 4}$ & 44,05 & 0,007 \\
\hline 'Fr-S' / 'NFr-S' & 0,3 & 0,47 & 1,06 & 1,125 & 2,47 & $\mathbf{0 , 8 4}$ & 16,33 & 0,0273 \\
\hline 'Fr-G' / 'NFr-G' & 0,12 & 0,16 & 0,12 & 0,125 & 0,15 & 0,008 & 0,025 & 0,88 \\
\hline
\end{tabular}




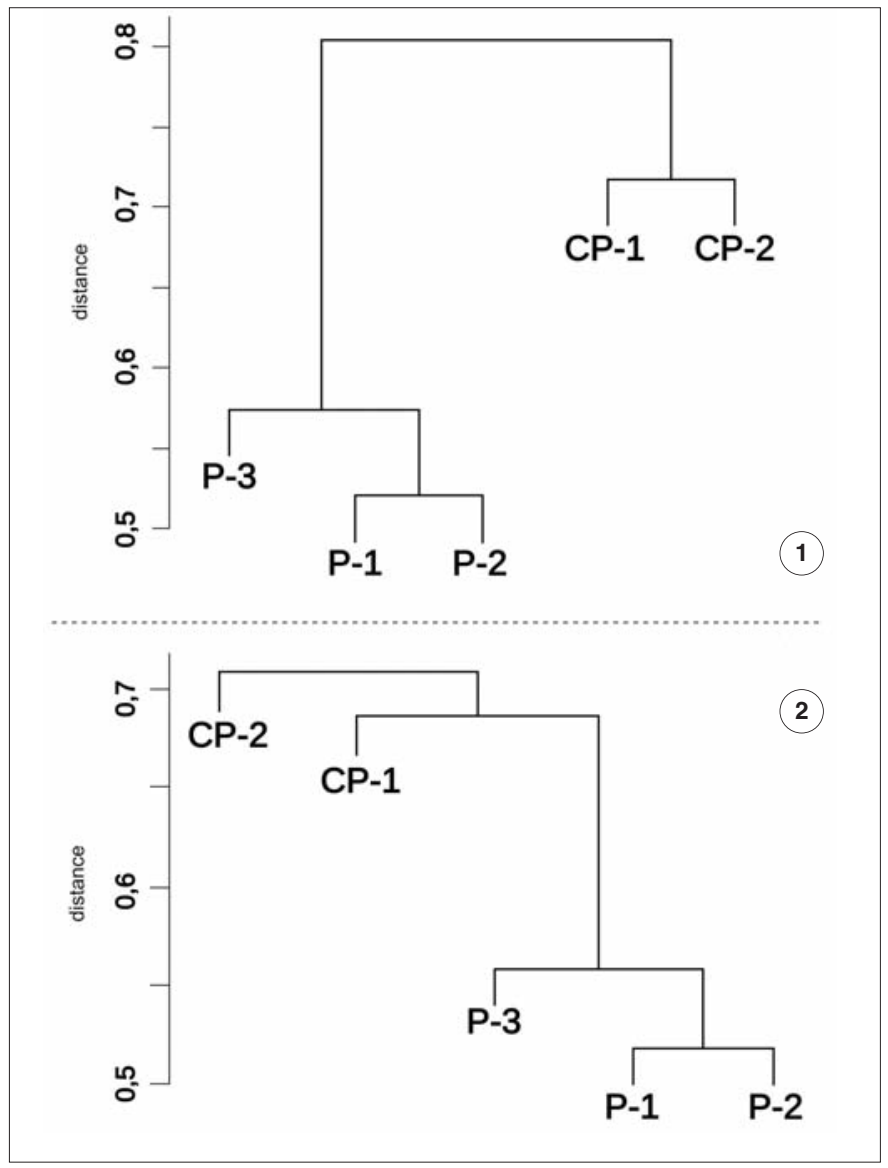

Fig. III - Similarity diagram of aphid communities on examined plots: $1-$ Ward method; Euclidean distance; 2 - UPGMA method; Euclidean distance.

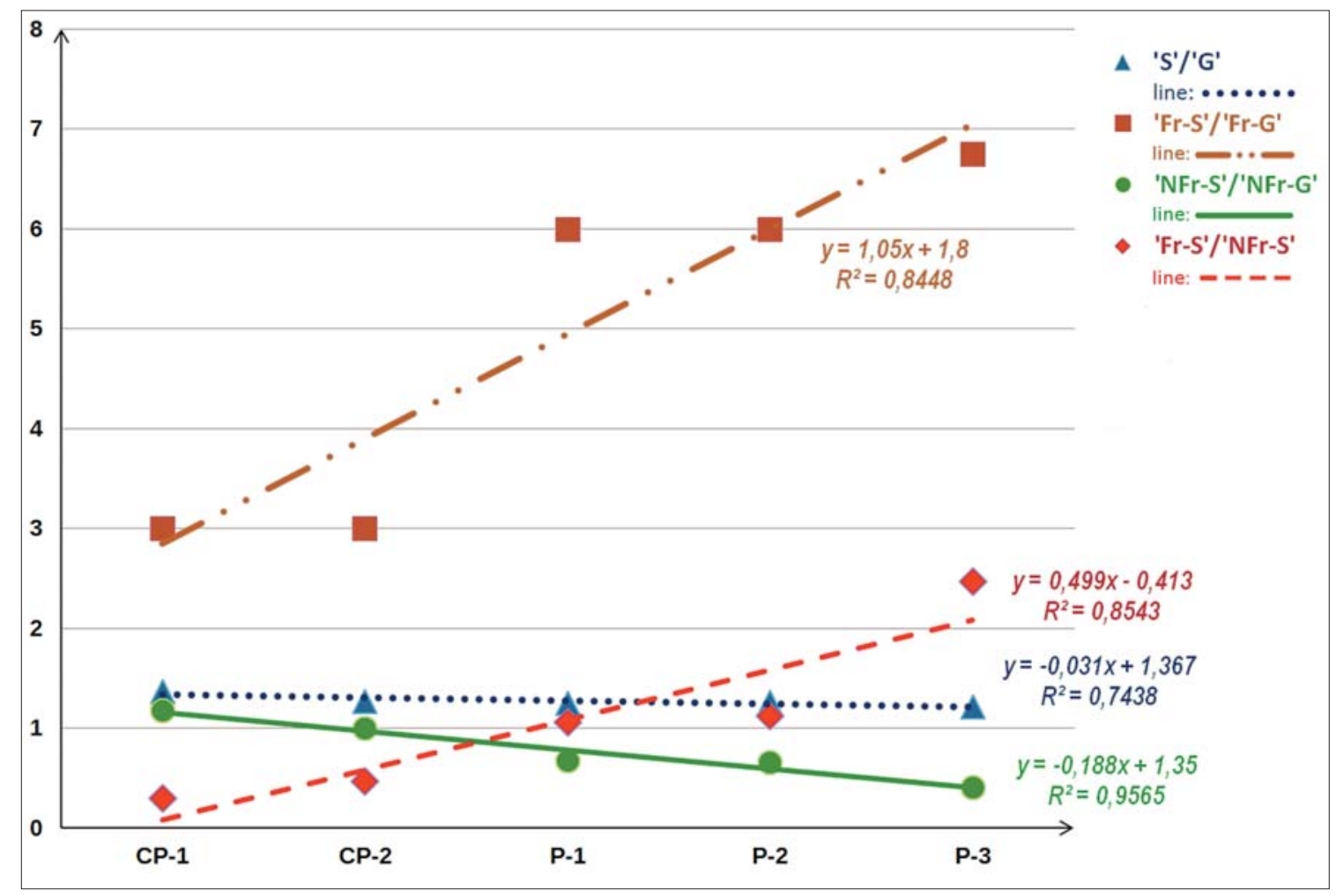

Fig. IV - Comparing the ratios of some ecological functional groups with their main trend lines (for values are significant at $P<0.05)$ : 'G' - generalists, 'Fr-G' - generalists in forest zones, 'NFr-G' - generalists in non-forest zones, 'S' - specialists, 'Fr$\mathrm{S}$ ' - specialists in forest zones, 'NFr-S' - specialists in non-forest zones. 


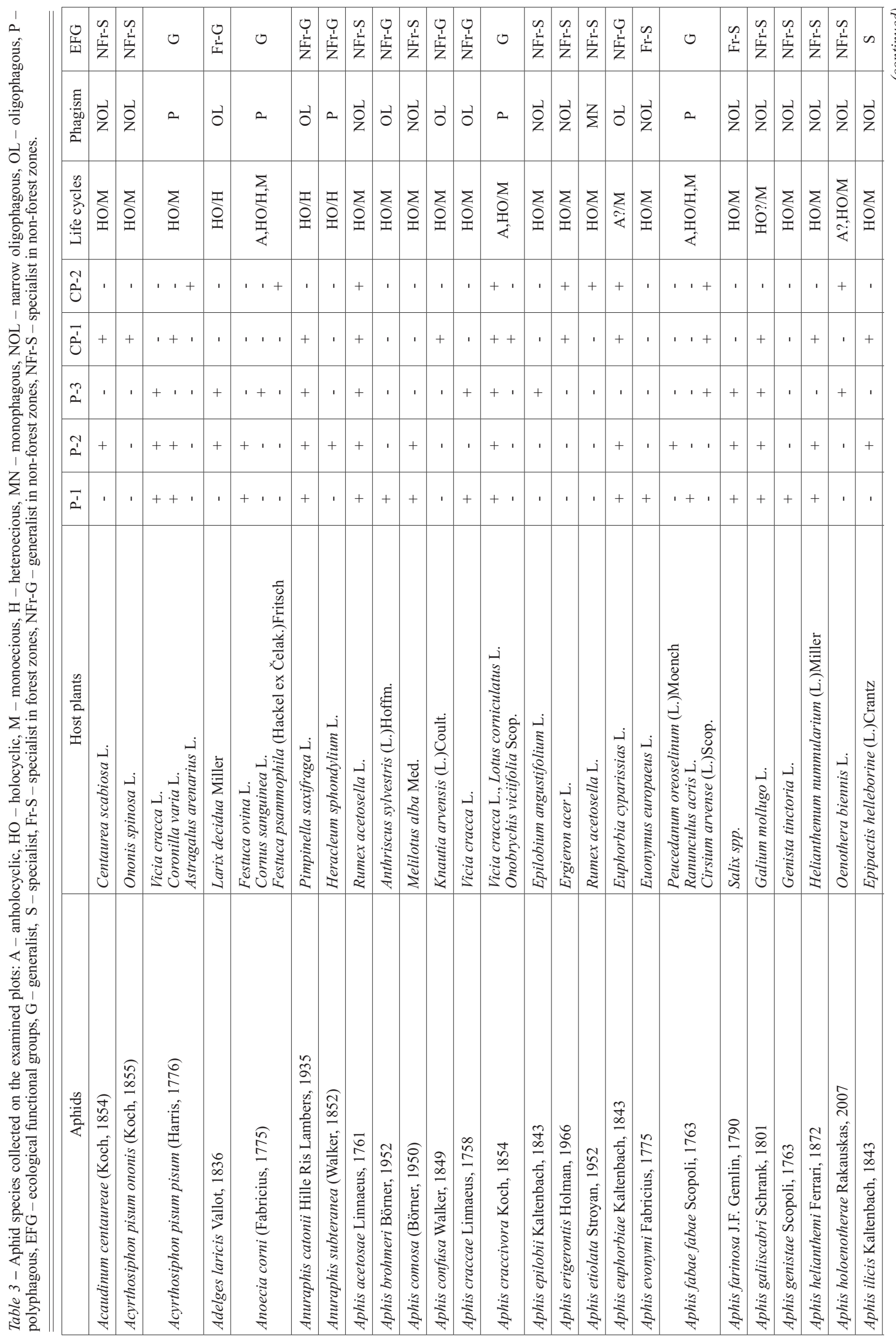




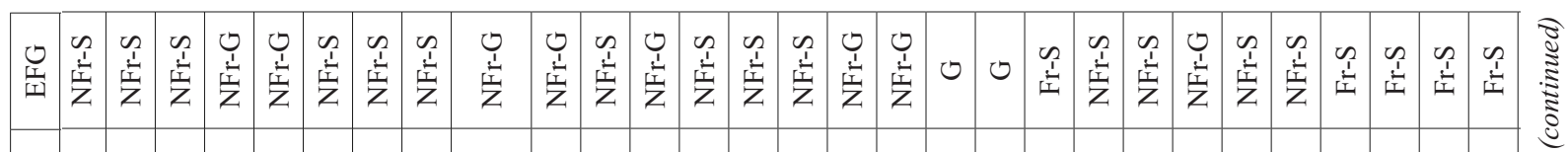

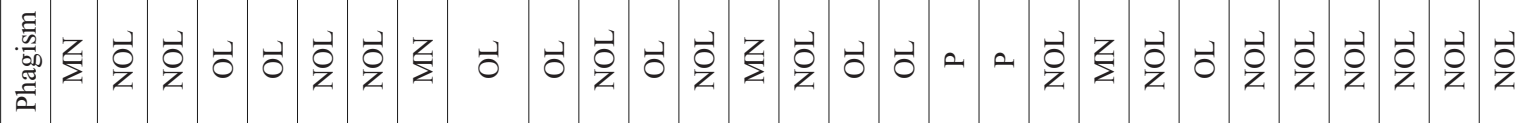

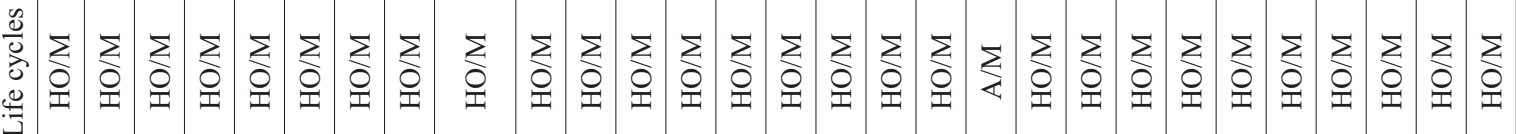
ปั่

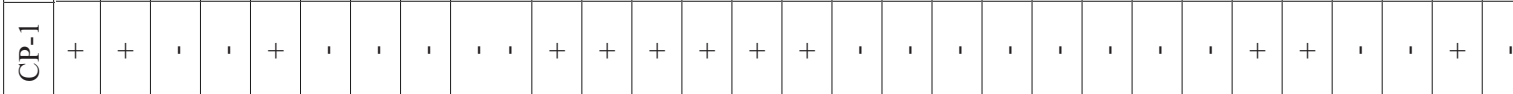
2 I $\overline{\mathbf{2}}++1+1+1+1+1$

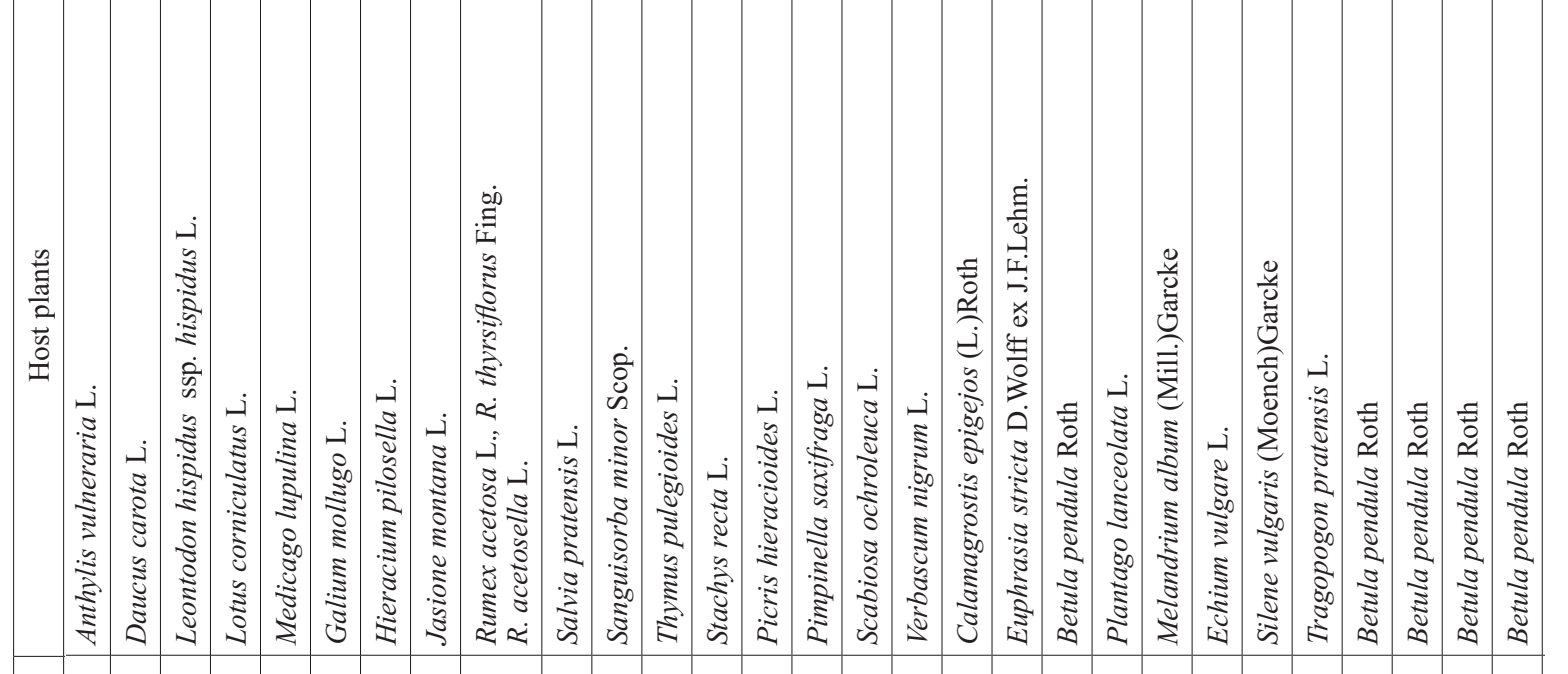

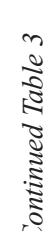

量

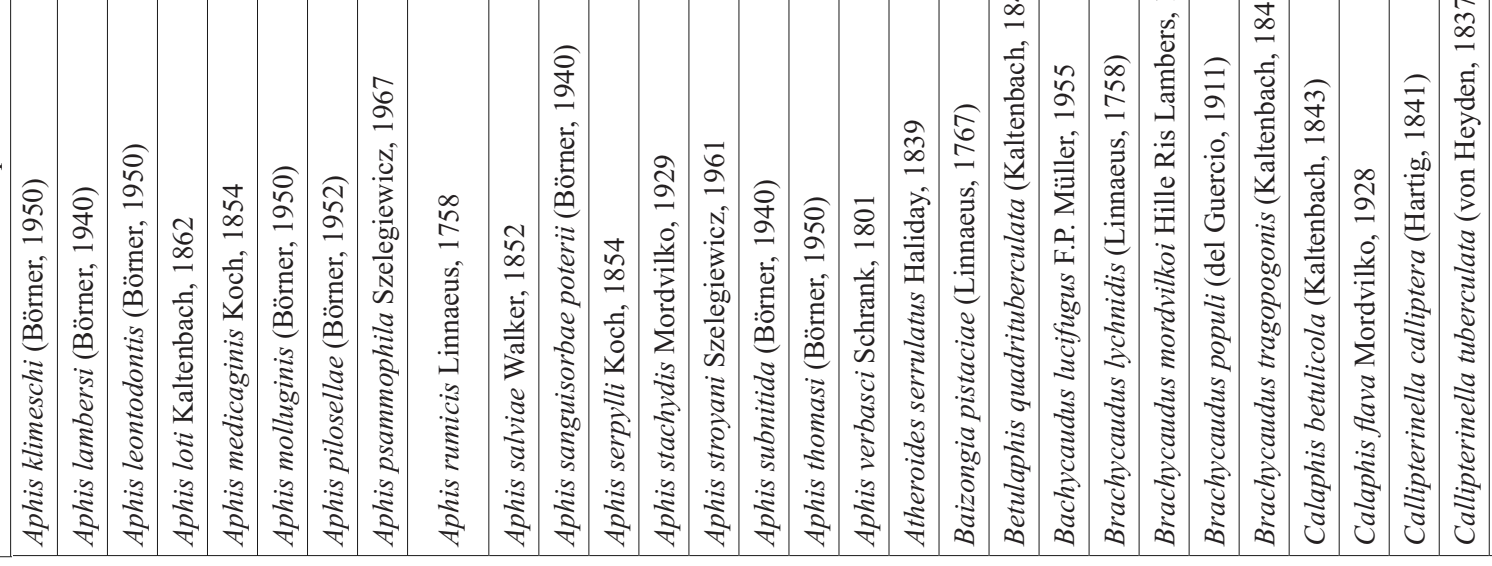

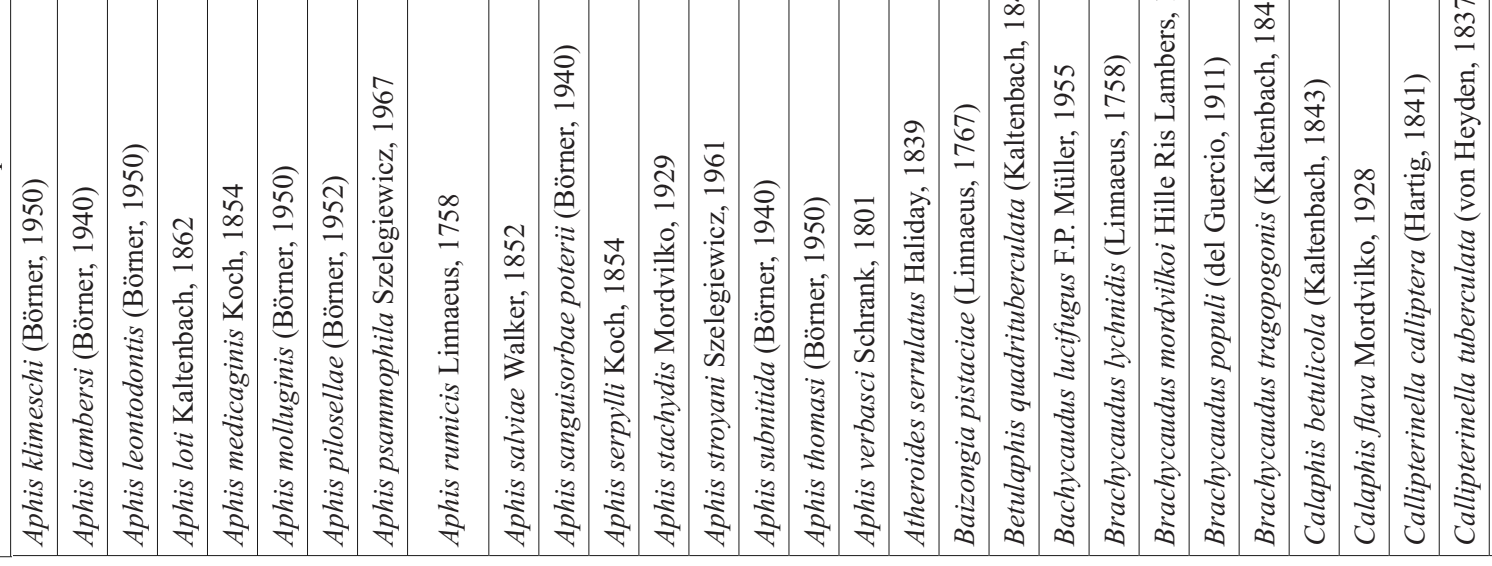




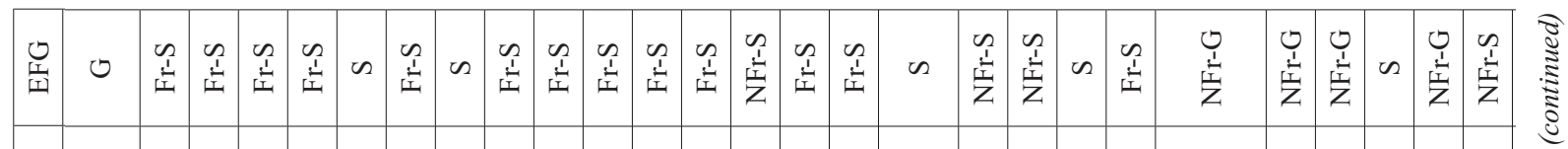

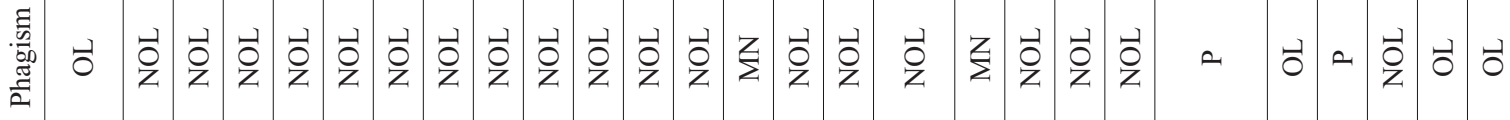

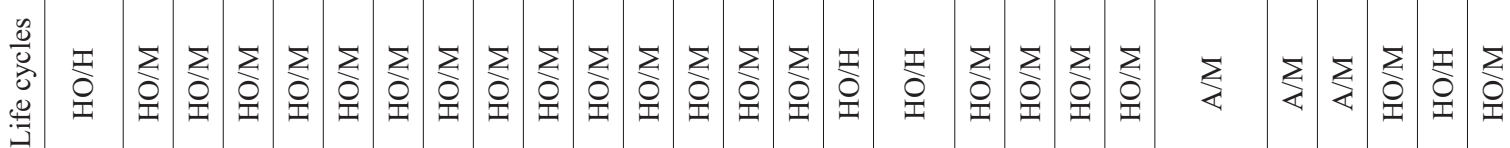

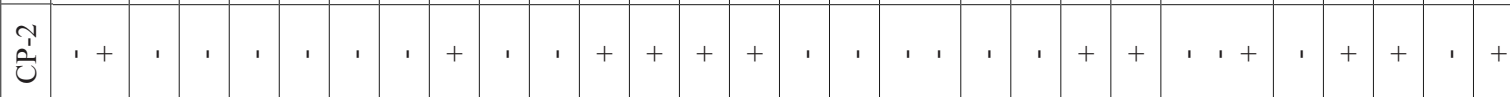

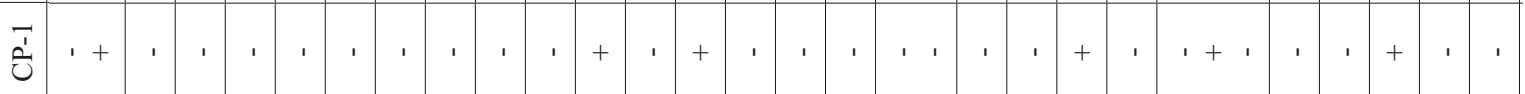

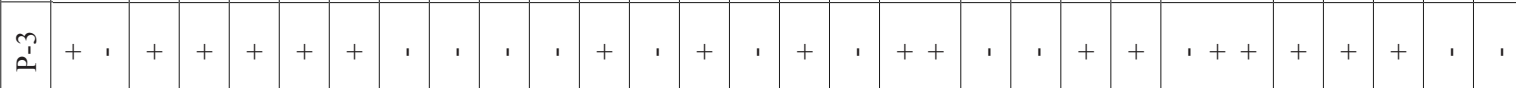
I $\overline{\mathbf{a}}+1 \cdot 1 \cdot 1+++1++$

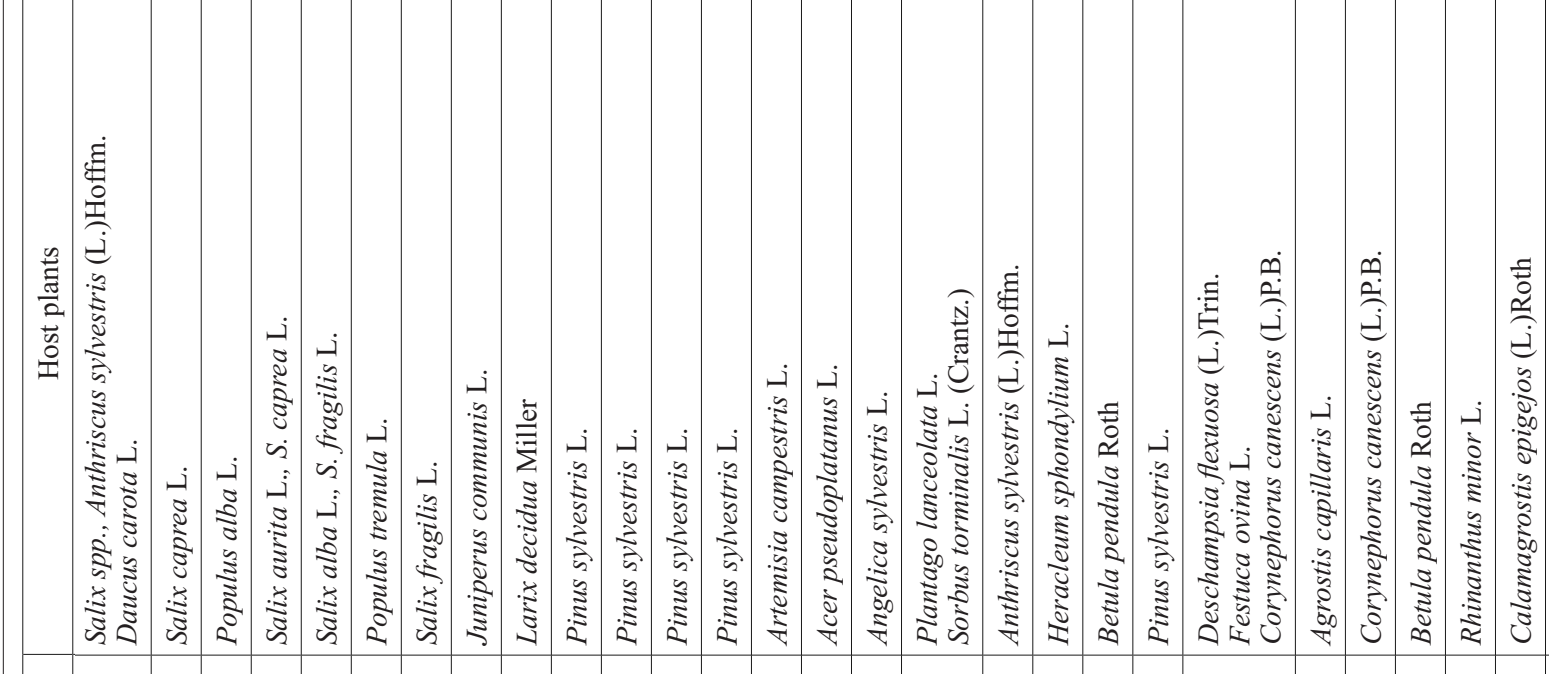

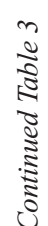

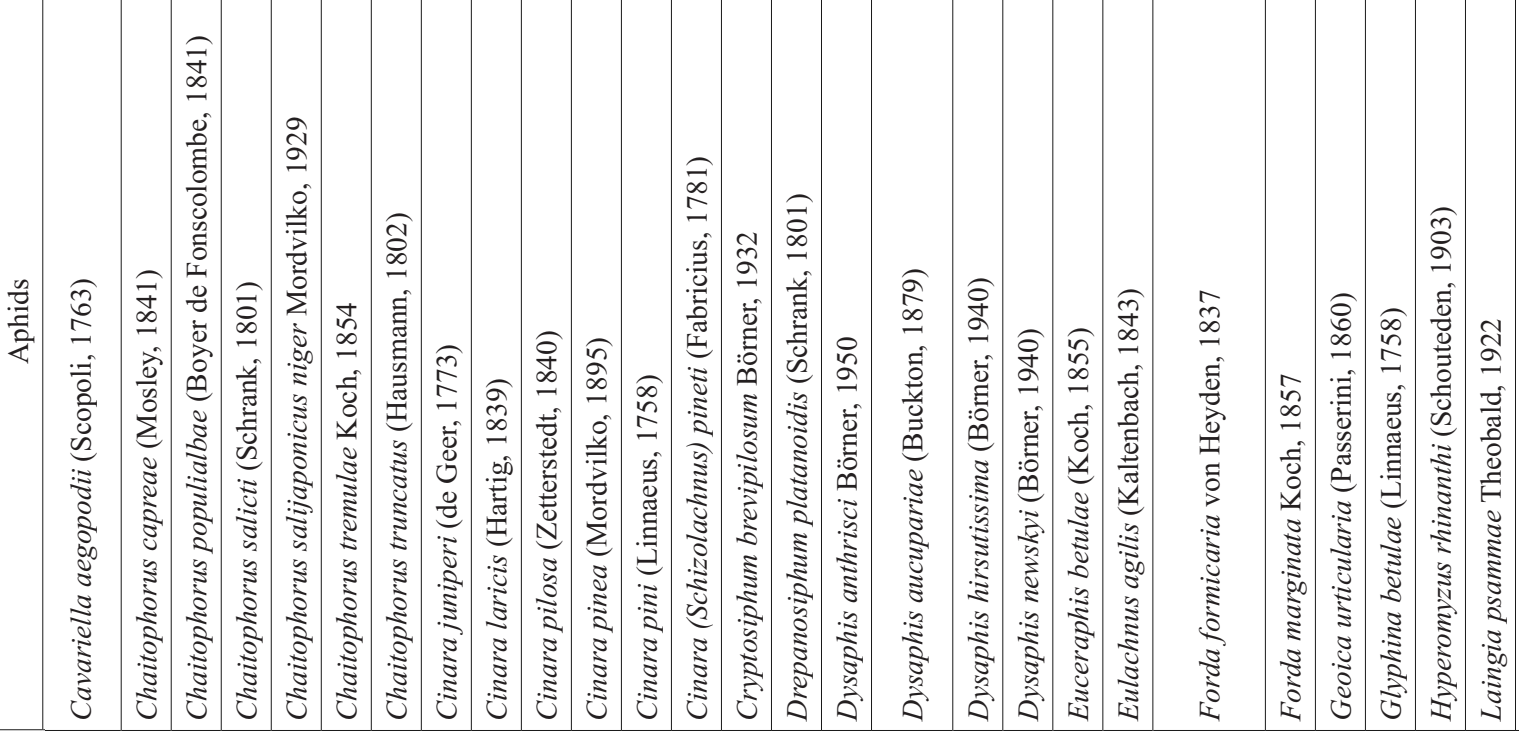




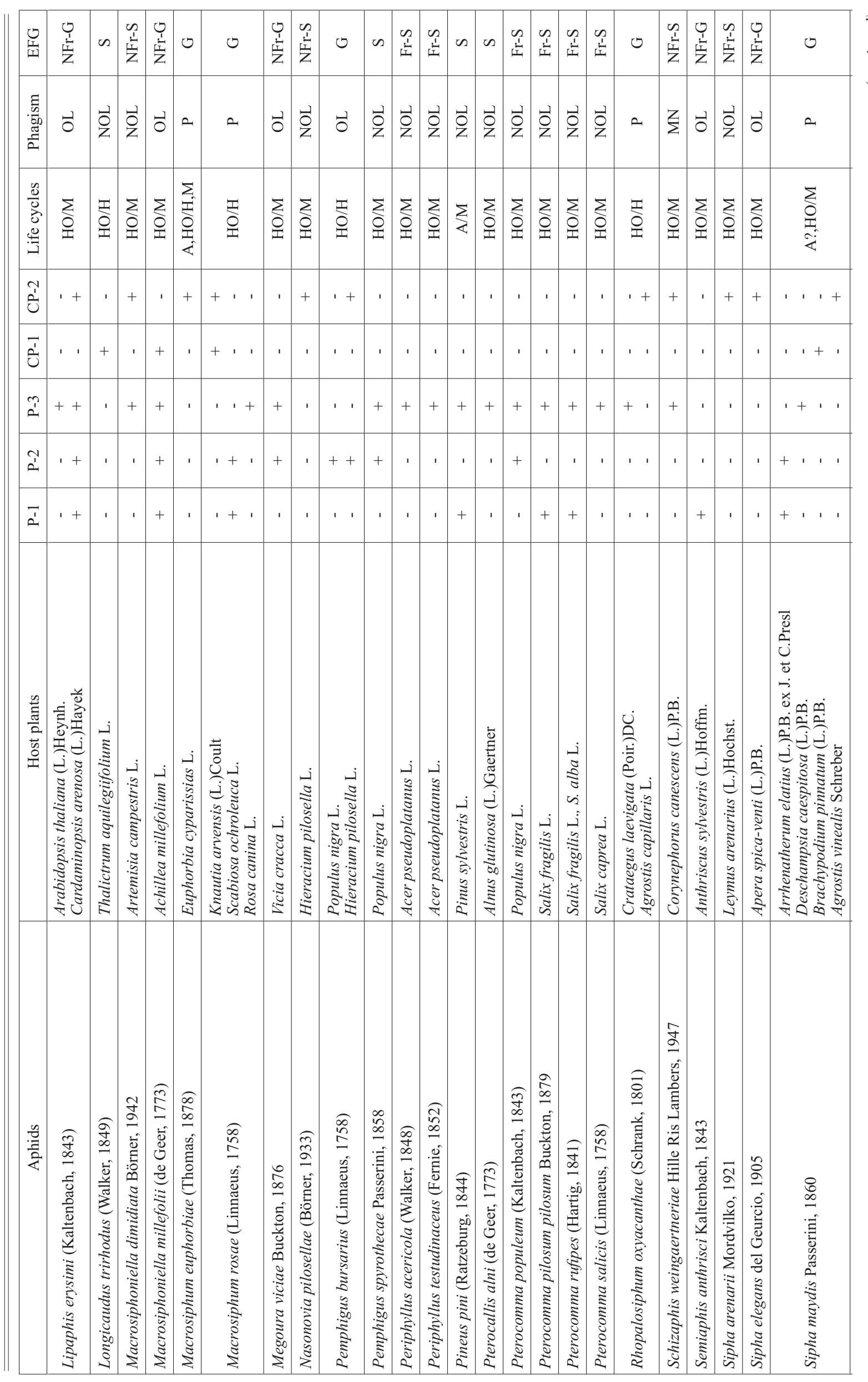




\begin{tabular}{|c|c|c|c|c|c|c|c|c|c|c|c|c|c|c|c|c|c|c|c|c|}
\hline $\begin{array}{l}0 \\
\underline{I} \\
I\end{array}$ & 0 & 䈉 & $\mid \begin{array}{l}0 \\
\vdots \\
\pm\end{array}$ & 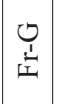 & $\infty$ & 0 & 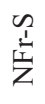 & $\begin{array}{l}0 \\
\frac{1}{1} \\
\frac{1}{Z}\end{array}$ & $\begin{array}{l}\text { 竞 } \\
\frac{1}{2}\end{array}$ & 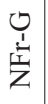 & $\begin{array}{l}0 \\
1 \\
\frac{1}{1} \\
Z\end{array}$ & $\begin{array}{l}0 \\
\text { 竞 } \\
\end{array}$ & $\begin{array}{l}\text { 号 } \\
\text { 苫 }\end{array}$ & $\mid \begin{array}{l}0 \\
\dot{1} \\
\dot{1} \\
z\end{array}$ & 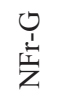 & 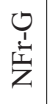 & $\begin{array}{l}\frac{2}{2} \\
\frac{1}{2}\end{array}$ & \begin{tabular}{l}
0 \\
$\vdots$ \\
\multicolumn{1}{|c}{} \\
$Z$
\end{tabular} & 足 & $\begin{array}{l}0 \\
\frac{1}{1} \\
\frac{1}{Z}\end{array}$ \\
\hline 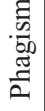 & $a$ & $\begin{array}{l}0 \\
z\end{array}$ & $\overrightarrow{0}$ & $\overrightarrow{0}$ & $\begin{array}{l}0 \\
\mathrm{z}\end{array}$ & 0 & o & 0 & ż & 하 & $\overrightarrow{0}$ & $\overrightarrow{0}$ & ô & 임 & 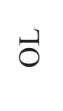 & $\overrightarrow{0}$ & ô & $\overrightarrow{0}$ & $z_{\Sigma}$ & $\overrightarrow{0}$ \\
\hline 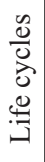 & 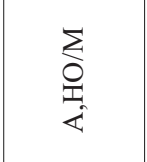 & $\sum_{\substack{i\\
}}$ & 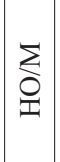 & $\begin{array}{l}\Sigma \\
0 \\
0\end{array}$ & $\begin{array}{l}\sum_{0} \\
0 \\
\end{array}$ & 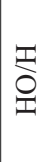 & 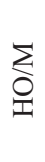 & 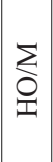 & 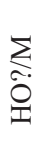 & 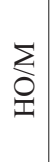 & $\mid \begin{array}{c}\Sigma \\
\vdots \\
0 \\
0 \\
<\end{array}$ & 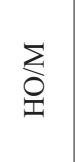 & 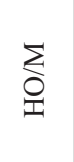 & 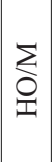 & 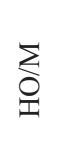 & $\begin{array}{l}\sum_{0} \\
\text { I }\end{array}$ & 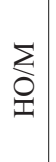 & $\begin{array}{l}\sum_{0} \\
0 \\
\end{array}$ & & \\
\hline $\begin{array}{l}\text { İ } \\
\text { Un }\end{array}$ & & & ' & ' & + & + & ' & ' & + & + & + & $1+$ & $1+$ & ' & ' & + & + & ' & ' & \\
\hline $\overrightarrow{\dot{u}}$ & + & + & ' & . & + & + & + & + & ' & + & ' & ' & 1 & ' & +1 & + & ' & + & + & \\
\hline$\stackrel{?}{2}$ & $11+1$ & ' & ' & ' & + & + & ' & + & . & + & ' & ' & $1+$ & + & ++ & + & ' & ' & . & + \\
\hline$\tilde{\Omega}$ & $1+1$ & ' & ' & ' & + & ' & ' & + & ' & + & ' & 1, & +1 & ' & +1 & + & ' & ' & . & + \\
\hline$\vec{\Xi}$ & $+1 \quad 1$ & & + & + & + & ' & & + & ' & + & & + & +1 & + & ' & + & I & ' & ' & \\
\hline
\end{tabular}
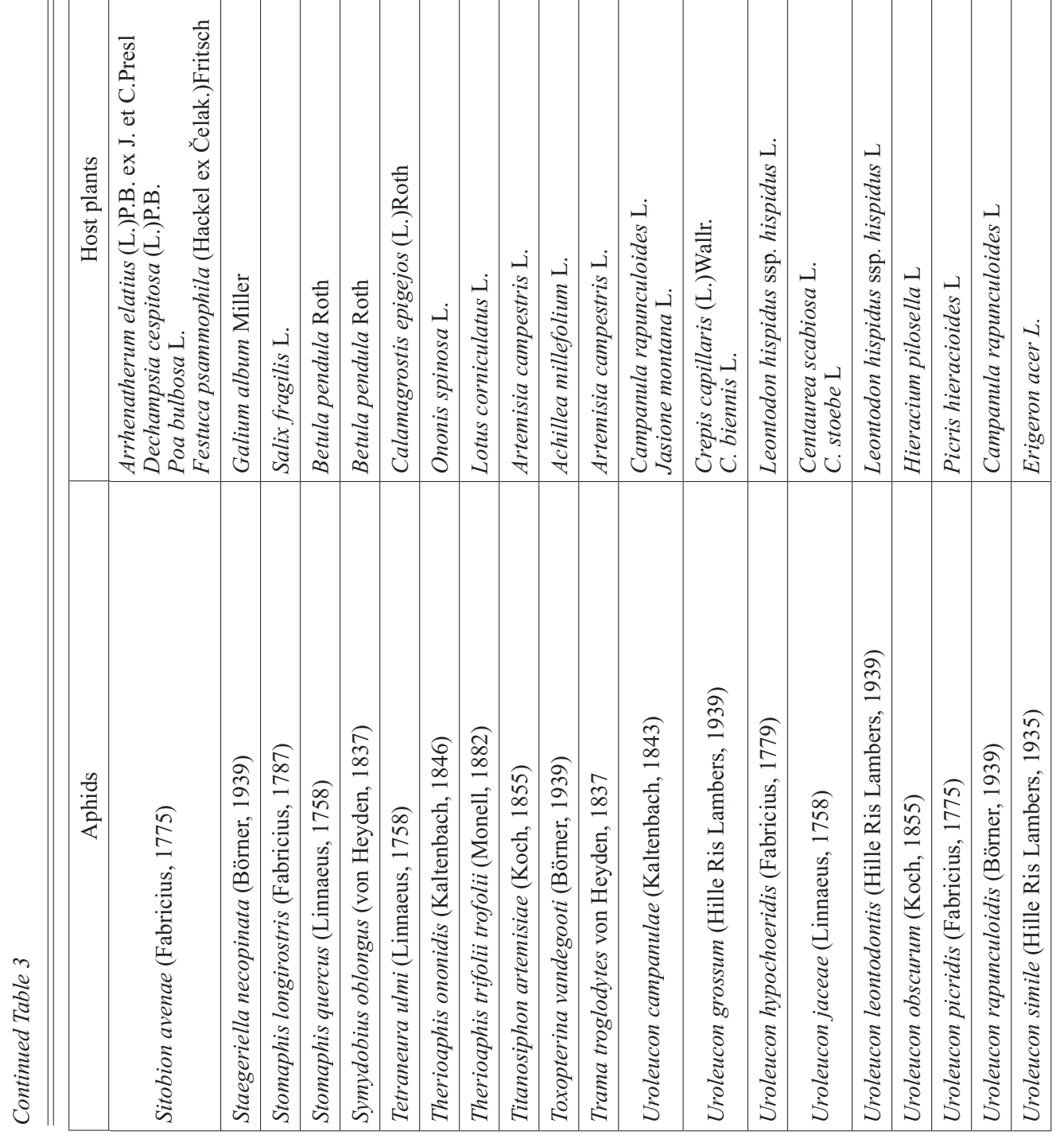


\section{Total \# of sp. = 125}

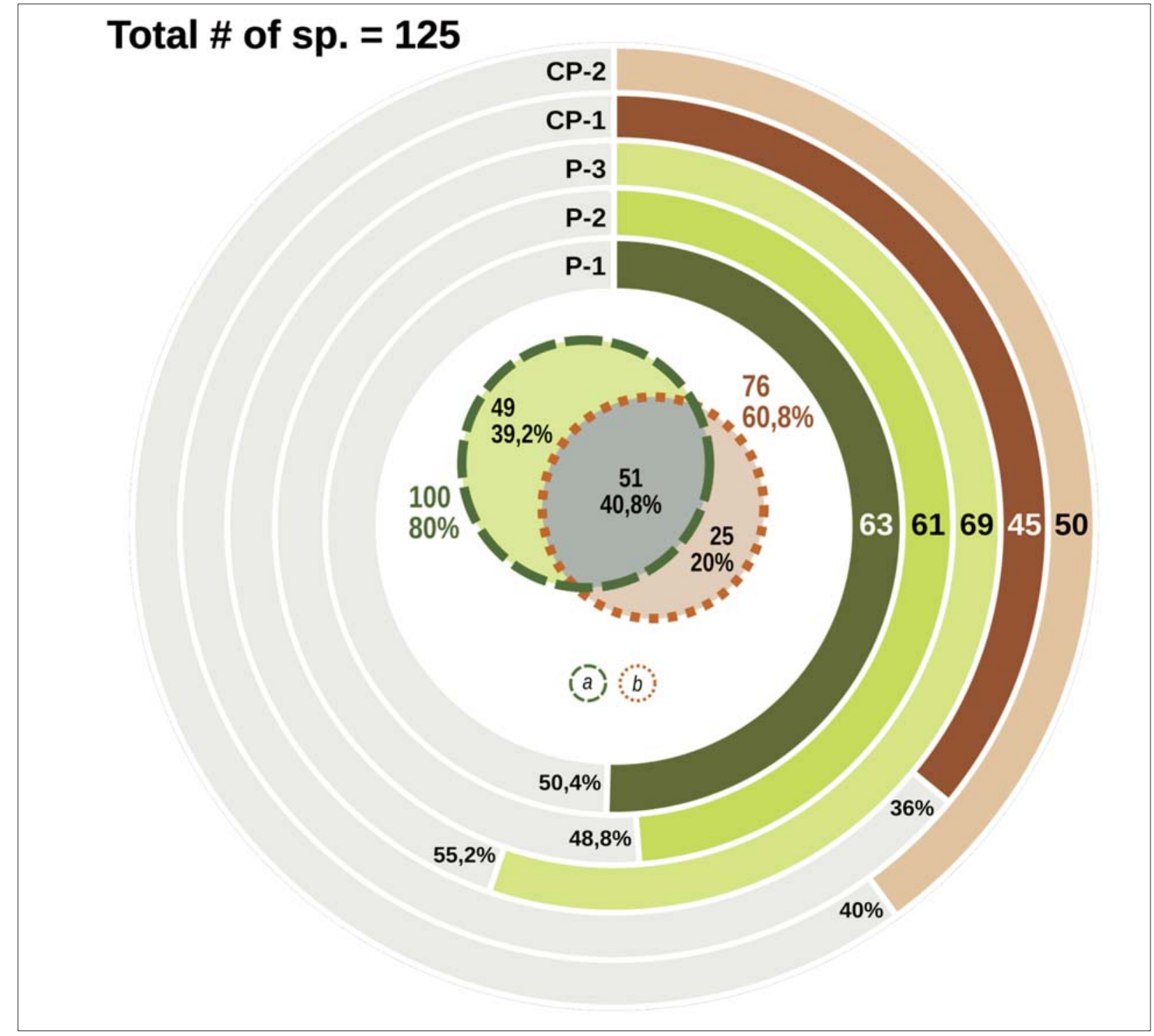

Fig. V - The number and percentage of aphid species found on the examined plots: a) Total on metalliferous areas; b) Total on control plots.

Out of all the aphid species only 48 were poorly specialized (14 polyphagous and 34 oligophagous). We recorded 39 non-specialized aphid species (eurytopic, i.e. species that have a wide range of ecological tolerance), in the metalliferous areas but only 9 on the control sites. The total number of specialized aphid species (stenotopic, i.e. species that have a narrow range of ecological tolerance) was 77 (68 were narrowly oligophagous, 9 were monophagous). We found 60 specialized aphid species in metalliferous areas, 34 of which (including 1 monophage) occurred only on the ' $\mathrm{P}$ ' plots. On the other hand, we recorded 13 specialized species (including 5 monophagous ones) only at the control sites.

The great majority of species found are monoecious (111); only 14 are heteroecious. 41 are associated with trees and shrubs (including 6 heteroecious ones), 29 of which occurred only in metalliferous areas, and only 1 at control sites. The others were found on both types of sites.

We found 84 species related to herbaceous plants (such plants are secondary hosts for 8 species); of these, 25 were found only in heavy metal areas, and 23 only at the control sites (the others were in both types of sites).
Of all the Aphidomorpha we recorded, 14 species are anholocyclic, and 7 are strictly asexual in Central Europe. The remaining ones occur as local anholocyclic populations or populations with a complete life cycle (holocyclic). Interestingly, we found Baizongia pistaciae from this group in rhizoid suckers of Euphrasia stricta - this is the first record of this aphid from semi-parasitic plants of the Orobanchaceae family.

We found a total of 41 aphid species on plants tolerating high concentrations of heavy metals (Table 1). Out of them 19 were poorly specialized species (10 of these are polyphagous). The others are narrowly oligophagous (20) or monophagous (2).

An analysis of species composition by using a fidelity index helped us to define diagnostic species (differential and characteristic species according to fidelity class). The material we collected does not include aphid species which could be considered differential for aphid communities related to metalliferous areas, however, we indicated 15 species which could be defined as characteristic, i.e. diagnostic species of this type of aphid community (Table 1). The class of accompanying species included 17 
taxa of Aphidomorpha listed as aphids connected with plants which tolerate high concentrations of heavy metals in soil (Table 1). We found 10 aphid species of indifferent class (Table 1).

Our analysis, which took into consideration the differentiating criteria of ecological functional groups ('EFG') of aphids, established ('P') a total of 57 (45.6\% of all the material) specialist species ('S') in the study area, 31 of which (24.8\%) from the 'Fr-S' subgroup and 26 (20.8\%) 'NFr-S' species. The less specialized species (generalists: ' $G$ ') totaled $45(36 \%)$; only 8 of these belonged to the 'Fr$\mathrm{G}$ ' subgroup $(6.4 \%)$, while the other $37(29.6 \%)$ were classified as 'NFr-G'. On the other hand, the control sites ('CP') yielded a total of $44(35.2 \%)$ species from the ' $S$ ' group (10 of which $(8 \%)$ were from the ' $\mathrm{Fr}-\mathrm{S}$ ' and 34 $(27.2 \%)$ from the 'NFr-S' subgroups) and 32 species from the ' $\mathrm{G}$ ' group (25.6\%) ('Fr-G' - 4 (3.2\%) and 'NFr-G' - 28 (22.4\%) respectively) (Fig. II).

The ratio of participations $\left(\mathrm{RP}_{\mathrm{EFG}}\right)$ of ' $\mathrm{S}$ ' group species to those of the ' $\mathrm{G}$ ' group was higher for ' $\mathrm{CP}$ ' at 1.375 than for ' $\mathrm{P}$ ' (1.26). A slightly higher $\mathrm{RP}_{\mathrm{EFG}}$ concerned the species which occurred only in 'CP' or only in ' $\mathrm{P}$ ' (2.57 and 1.88 respectively) (Fig. IV). The situation is slightly different in particular 'EFG' subgroups. For 'NFr-S' to 'NFr-G' the ratio of participations was as above, i.e. higher for ' $\mathrm{CP}$ ' than for 'P', while for ' $\mathrm{Fr}-\mathrm{S}$ ' to 'Fr-G' it was the opposite, i.e. higher for ' $\mathrm{P}$ ' than for ' $\mathrm{CP}$ '. Also the ratios of 'Fr-S' to 'NFr-S' was higher for ' $\mathrm{P}$ ' (Fig. IV). The detailed data for the numerical and percentage shares of aphids from particular ' $E F G$ ' in the study areas and their $\mathrm{RP}_{\mathrm{EFG}}$ are listed in Table 2. To facilitate interpretation we have also presented them graphically (Figs. II, IV).

Statistical analysis of similarity between the aphid communities of particular plots ('Ward' and 'UPGMA') demonstrated a greater similarity between the aphid communities in ' $\mathrm{P}$ ' than between these and those from the control ' $\mathrm{CP}$ ' plots. This constitutes evidence for the different character of aphid communities associated with heavy metal grasslands (Fig. III).

\section{DisCUSSION}

Seeing constant changes to earth's biocoenoses, particularly those related to human impact (anthropopressure), it is necessary to learn the current state of plant life and wildlife richness. This will help to evaluate profits or losses to this richness in future (change directions) and its proper management in order to conserve the status quo of the world's gene pool, or at least reduce the possible losses. In European landscape semi-natural dry grasslands can serve as an example of such exceptional and sitll insufficiently known environments. However, as proven by studies, despite extremely unfavourable conditions, these habitats are characterised by a significant species richness of flora and fauna (e.g. CHMURA et al., 2013; OsIADACZ and HAŁAJ, 2015; ÖCKINGER et al., 2006); they even may constutite biodiversity hotspots (CREMENE et al., 2005). Such habitats are also calaminarian grasslands, in which plant species coming from dry calcareous grasslands (xerothermic grasslands) have a significant share (NowAK et al., 2015).

\section{APHID COMMUNITIES AS DEFINED BY FIDELITY CLASSES} AND QUESTION OF DECISIVE CRITERIA

Aphid diversity of heavy metal grasslands is high, as we found there 125 aphid species (Table 3), most of which are stenotopic ones. Moreover, excessive concentration of heavy metal in soil do not adversely influence the number of species of such areas. Thus species richness of aphids is characteristic for control plots which represent dry grasslands (dry calcareous and sandy grasslands) formed on soil of similar physical and chemical parameters, but without excessive concentration of heavy metals (Table 3, Fig. V). Out of 125 aphis species we found (Table 3 ) as many as 41 fed on plants which tolerate high concentration of heavy metal in soil (Table 1).

While characterising species composition of the studied aphid community of heavy metal grasslands, interesting species which occur solely in a particular habitat even though their host plants are common in other habitats were found. Analysing the level of relationship between an aphid species within the studied habitat (based on a host plant, particularly its diagnostic value for a relevant plant community) enabled determination of fidelity classes for aphid species (Table 1). This in turn helped to define diagnostic species (fidelity classes 3 and 4 ) and prove a distinctive character of such an aphid community.

We found 15 aphid species to be diagnostics ones for the aphid communities analysed (Table 1). In all the 5 plots, 5 aphid species were commonly present: Aphis lambersi, Brachycaudus populi, Cinara pinea, Uroleucon leontodontis, Cinara (Schizolachnus) pineti (Table 1). Characteristic species of aphid community related to all the types of dry grasslands include Aphis lambersi, Brachycaudus populi and Uroleucon leontodontis - species narrowly oligophagous, connected with herbaceous plants of all the treeless plant communities. Their host plants (Daucus carota, Silene vulgaris and Leontodon hispidus respectively) have their optimum occurrence in other plant communities and are characteristics of semi-natural meadows (of MolinioArrhenatheretalia R.Tx. class). Interestingly, A. lambersi and $U$. leontodontis in meadows are reported only sporadically (CZYLOK et al., 1988) and most often not at all (e.g. CzYLOK and WoJCIECHOWSKI, 1987; WoJCIECHOWSKI and WoźNICA, 1989; BorowIAK-SOBKOWIAK et al., 2008). These species are much more often recorded in sandy or dry calcareous grasslands (e.g. HAŁAJ and WoJCIECHOWSKI, 1996; DEPA and WoJCIECHOWSKI, 2009; OsIADACZ and HaŁAJ, 2015), or possibly, more rarely, in drier forms of synanthropic plant communities (mainly of Onopordietalia acanthi Br.-Bl. et R.Tx. 1943 em. Görs 1966 order) (DEPA and MRÓz, 2012). $C$. pinea and $C$. (S.) pineti occurred in all the five plots (Table 1,3), but we rarely recorded them. These species feed only on pines (especially Pinus sylvestris) and are characteristic for aphid communities of pine forests of Dicrano-Pinion Libb. 1933 alliance (SzELEGIEWICZ, 1976). There they reach a high stability and are often dominants or subdominants (e.g. CZYLOK, 1983; HaŁaJ and WOJCIECHOWSKI, 1997; DURAK and WOJCIECHOWSKI, 2005). Despite their being narrow oligophages, we considered them to be accompanying species, because both them and the other species found on the pine (Cinara pini, C. pilosa, Eulachnus agilis and Pineus pini) often occur in habitats with human pressure (anthropogenic habitats) (e.g. BOROWIAK-SOBKOWIAK and WILKANIEC, 2010; BUDZIŃSKA and GosZCZYŃSKI, 2010). The same Dysaphis aucupariae occurred in all the plots with heavy metal plants (P-1, P-2, P3 ) and was not found in control plots (CP-1, CP-2). We could not consider it to be a differential species, as it is heteroecious and its main primary host plant, Sorbus torminalis, has been introduced in all the study region and is numerous in parks and street greenery. The remaining 12 species regarded as diagnostic and characteristic for aphid communities of metalliferous areas (Acaudinum centaureae, Aphis euphorbiae, A. galiiscabri, A. helianthemi, A. klimeschi, A. leontodontis, A. molluginis, A. pilosellae, A. 
subnitida, Brachycaudus lucifugus, Nasonovia pilosellae, Uroleucon obscurum - Table 1) are more often reported, albeit occur rather locally (HEIE, 1986; OsIADACZ and HA£AJ, 2009; Nieto Nafria et al., 2005). Some of them (i.e. $A$. klimeschi, A. subnitida, B. lucifugus) are rare for Europe (Holman, 2009) and are specialized, stenotopic species, which prefer particular types of dry, warm habitats (OSIADACZ and WOJCIECHOWSKI, 2008), and can be classified as specialists of treeless zones ('NFr-S'), even as type K strategy specialists (OSIADACZ and HAŁAJ, 2011, 2015; HAEAJ and OSIADACZ, 2014).

In our opinion, for some of these species the trophic criterion (i.e. host plants) and abiotic factors, such as temperature and humidity, are not the most significant ones, as the decisive criterion can be soil morphology. For instance B. lucifugus, occurred only in P-2 and CP-2, although Plantago lanceolata, its host plant, occurred quite frequently at all the plots with similar microclimatic conditions. In these sites the structure of upper soil layers is skeletal and fine-grain, which means rather loose and aerated. On the other hand, we recorded $A$. centaureae and A. klimeschi only in $\mathrm{P}-1$ and $\mathrm{CP}-1$, the plots where the upper soil layers are more compact and much less aerated. Other species (A. subnitida, A. helianthemi, A. pilosellae) were found in P-1, P-2 and CP-1 or CP-2 (they did not occur in $\mathrm{P}-3$ ). Another one (A. leontodontis) was reported only from P-3, where the upper soil layer is skeleton-like and coarse-grained. The soil morphology was not important for some aphid species, namely those which we found in all the plots (i.e. A. lambersi, B. populi, $U$. leontodontis).

Such habitat preferences concern not only aphids feeding on plants with various level of relation to metalliferous areas (Table 1), but also species generally recorded throughout the study (Table 3). For example, Aphis etiolata, Cryptosiphum brevipilosum, Nasonovia pilosellae and Sipha arenarii occurred only on sandy grassland (CP-2), whereas Aphis stroyani, A. sanguisorbae poterii, A. stachydis, Staegeriella necopinata and Therioaphis ononidis on dry calcareous grassland (CP-1) (Table 3). Although Angelica sylvestris grew in all the plots, the aphid related to this plant - Dysaphis anthrisciwas found only in metalliferous areas (P-1, P-2, P-3). Other species (e.g. Aphis galiscabri, A. ilicis) belonged to aphid communities related both to heavy metal grasslands $(\mathrm{P}-1, \mathrm{P}-2, \mathrm{P}-3)$ and dry calcareous grassland (CP-1), however, absent on sandy grassland (CP-2). (Table 3$)$. The case was opposite for Macrosiphoniella dimidiata and Schizaphis weingaertneriae, which were not recorded on dry calcareous grassland (CP-1).

APHID COMMUNITIES AND ECOLOGICAL FUNCTIONAL GROUPS

Here we use for the first time the concept of ecological functional groups ('EFG') aphid communities analysis. It proved the dominance of aphids of specialist groups ('S') on all the study plots (Fig. II). This had been expected, as the definite majority of recorded species is closely related to their host plants. This is confirmed also by other aphidological papers which include notes on the biology of aphid species of interest to us (e.g. HeIE, 1986; NiETo NAFrIA et al., 2005; BLACKMAN and EASTOP, 2015). The determining of the number or the percentage of aphid species of particular ' $\mathrm{EFG}$ ' in aphid communities is interesting; the comparing of these groups ratios $\left(\mathrm{RP}_{\mathrm{FFG}}\right)$ in forming aphid communities related to particular plant communities is no less interesting and also significant. So far no such studies have been conducted. Only sole paper touched upon the problem
(DURAK et al., 2011). Those authors report that stable plant communities, formed in conditions with comparatively stable and homogenous impact of abiotic factors, lead to equally stable aphid communities in which stenotopic species of specialist group ('S') play the main role. However, in the ecotone zone (fringe communities) and aphid communities related to synanthropic communities (i.e. unstable habitats, subject to constant changes) the number of specialist species is decreasing in favor of general ones (i.e. species of wide ecological tolerance - ' $G$ '). This conclusion can be corroborated by an analysis the lists of aphid species in papers which address both natural (e.g. HAŁAJ, 1993; DURAK and Wojciechowski, 2005; Osiadacz and WieczoreK, 2007) and cultural (anthropogenic) landscapes, including agrarian (e.g. WiLKANIEC et al., 1999; CichOcKA and LUBIARZ, 2010; KIRCHNER et al., 2013) and urban zones (e.g. RUSZKOWSKA and WILKANIEC, 2002; OsIADACZ and WIECZOREK, 2003; WILKANIEC et al., 2012). The presence of specialists is clearly visible in urban landscapes in forest zones ('Fr-S'), while at the same time a considerable fall in specialist ones in non-forest zones ('NFr-S') and an increase of aphid species number which are not generalist in nonforest zones ('NFr-G'). This is mainly due to artificial introduction of various tree and shrub species in the landscape, which are aphid host plants.

The assumption, that depending on the human pressure factors the proportion of aphid species of particular ecological functional groups ('EFG') changes in aphid communities structure is corroborated by our study. We found the dominance of specialists (' $S$ ') in all the aphid communities (Table 2; Fig. II). However, an increased share of specialists in forest zones ('Fr-S') of aphid communities in heavy metal plants (P-1, P-2, P-3) and at the same time a decrease in the share of generalists in forest zones ('Fr-G') and specialists in non-forest zones ('NFr-S') is visible in the trend lines (Fig. III). A significantly higher than expected number of specialists in forest zones ('Fr-S'), which also make up aphid communities in dry grasslands (HALAJ and WoJCIECHOWSKI, 1996; OsIaDACZ and HataJ, 2015) can be explained with old and incorrectly conducted land reclamation aimed only at a fast forestation of the area. Currently, after some of the plots (P-1, P-2) were included in the 'Nature 2000' network, the other principles of active protection were introduced there. These comprised mainly a gradual removal of trees and shrubs introduced there artificially and thus creating favorable conditions for unique metalliferous flora (KAPUSTA et al., 2010; KowOLIK et al., 2010). The plot P-3 is not subject to such treatment, which is reflected in its aphid community by the highest share of specialists in forest zones ('Fr-S') in comparison with the specialists in non-forest zones ('NFr-S') and generalists in forest zones ('Fr-G').

Valuable information on the condition of aphid communities can be provided by the ratio of participations $\left(\mathrm{RP}_{\mathrm{EFG}}\right)$ of specialists in non-forest zones ('NFr-S') to generalists in non-forest zones ('NFr-G'). In unstable aphid communities (subjected to human factor) the above proportion is less than $1\left(\mathrm{RP}_{\mathrm{EFG}}\right.$ 'NFr-S' to 'NFr-G' $\left.<1\right)$. On the other hand, rather stable aphid communities yield its value above $1\left(\mathrm{RP}_{\mathrm{EFG}}\right.$ 'NFr-S' do 'NFr-G'> 1$)$ (Table 2, Fig. II). A The analysis of at least some ratios $\left(\mathrm{RP}_{\mathrm{EFG}}\right)$ can help to answer the question of the condition of aphid community. If such an analysis is conducted every few years (e.g. 4-5), it could indicate changes in aphid communities, and thus evaluate the increased or decreased level of human impact. 
SIMILARITIES OF DISTINGUISHED APHID COMMUNITIES

Although in the studied plots disturbances caused by human activity are observed, there is no doubt that characteristic aphid communities were formed there. Each community is characterised by a slightly different species composition, ratios of ecological functional groups and each can be differentiated from the others based on the presence of locally characteristic species, i.e. diagnostic ones. Aphid species structure of metalliferous areas indicates that these plots are inhabited by species typical of aphid communities in dry calcareous and sandy grasslands and, to a lesser extent, other ones (pine forests of Dicrano-Pinion alliance or meadows of Molinio-Arrhenatheretalia class). As a result new aphid communities are formed of individual combination of species.

Such a conclusion is confirmed by a similarity analysis which we conducted on the basis of species composition of the studied plots. Aphid communities formed in metalliferous areas (P-1, P-2, P-3) show significantly higher similarities among each other (Fig. III) than between them and aphid communities of dry calcareous grassland (CP-1) and sandy grassland (CP-2) (Fig. III). On the other hand, significant similarities of all the studied aphid communities of all plots (comparatively small distances in the dendrogram - Fig. III) confirm the forming of a wider circle of aphid communities associated with all types of dry grasslands (in a wide sense) (HAŁAJ and WoJCIECHOWSKI, 1996; OsIADACZ and WoJCIECHOWSKI, 2008). The individual character of aphid community of P-3 plot can be explained with a different origin of this habitat (it is a slag heap and not a mining spoil heap) and a longer distance from the nearest centres of aphid communities of dry calcareous and sandy grasslands (HAŁAJ and WOJCIECHOWSKI, 1996; HAŁAJ, 1999; Osiadacz and Wojciechowski, 2008). Such a distance from natural ranges and centers of occurrence of particular aphid species, according to the theory of environmental islands and metapopulation, may hinder colonisation and survival of the individuals (i.e. species) newly arrived in new areas (e.g. HAILA, 2002; HANSKI and OvASKAINEN, 2003; LOSOS and RiCKLEFS, 2009).

Summing up, the results we have obtained and presented give grounds for claiming that metalliferous areas help to form species-rich, characteristic aphid communities associated with heavy metal grasslands. By species richness we mean that the number of aphid taxa in these communities is comparable with those of dry calcareous or sandy grasslands. This species richness may indicate that excess concentrations of heavy metals in the soil do not limit the number of aphid species in the aphid communities there; conversely, the specific nature of these communities means that they consist of a mixture of species which have arrived (and settled) from other habitats. Their main components are the species from dry calcareous and sandy grassland aphid communities. Like these two aphid communities, the one associated with heavy metals grasslands derives its equally unique character from nonforest zone specialist aphid species, also rare at the European scale. The ratios between this species group and other ecological functional groups of aphids (particularly non-forest generalists) can define the condition of an aphid community (and thus the habitat they are associated with). If they were calculated at specific time intervals (e.g. several years), they could be indicative of changes in the aphid communities and define their directions, and thus could become a very useful tool for monitoring habitat condition.

\section{ACKNOWLEDGEMENTS}

We are very grateful to Dr. Damian Chmura (Institute of Environmental Protection and Engineering, University of Bielsko-Biala, Poland) for help with the statistical analysis and to Dr. Jacek Szwedo (Department of Invertebrate Zoology and Parasitology, University of Gdansk, Poland) for discussions during preparation of this paper and linguistic corrections. We would also like to thank Anonymous Reviewer for all valuable remarks and suggestions on the manuscript.

\section{REFERENCES}

BABCZYŃSKA-SENDEK B., 2005 - Problemy fitogeograficzne i syntaksonomiczne kserotermów Wyżyny Slaskiej. Wydawnictwo Uniwersytetu Śląskiego, Katowice, 237 pp. (in Polish)

Baker A.J.M., ERnst W.H.O., VAn der Ent A., Malaisse F., Ginocchio R., 2010 - Metallophytes: the unique biological resource, its ecology and conservational status in Europe, central Africa and Latin America. In: Ecology of Industrial Pollution, Batty L.C. \& Hallberg K.B. Ed., Cambridge University Press, British Ecological Safety, Cambridge, pp.7-40.

BECKER T., 2012 - VIOLETEA - heavy metal grasslands. In: Vegetation databases for the 21st century, Dengler J., Oldeland J., Jansen F., Chytrý M., Ewald J., Finckh M., Glöckler F., Lopez-Gonzalez G., Peet R.K. \& Schaminée J.H.J. Ed., Biodiversity \& Ecology, 4: 325.

BLACKMAN R.L., EASTOP V.F., 2015 - Aphids on the World's Plants. An online identification and information guide. $<$ http://www.aphidsonworldsplants.info/>, accessed $1^{\text {st }}$ January 2016.

Borowiak-SobKowiak B., DURAK R., GrZElaK M., 2008 Aphids (Hemiptera, Aphidoidea) on meadow communities from the Molinio-Arrhenarethetea class. - Aphids and Other Hemipterous Insects, 14: 51-61.

Borowiak-Sobkowiak B., Wilkaniec B., 2010 Occurrence of aphids (Hemiptera, Aphidoidea) on tree and shrubs in Cytadela Park in Poznań. - Aphids and Other Hemipterous Insects, 16: 27-35.

BraUn-Blanquet J., 1964 - Pflanzensoziologie: Grundzüge der Vegetationskunde. Springer, Wien-New York, 865 pp.

BUDZIŃSKA L., GOSZCZYŃSKI W., 2010 - Coniferous plants in the Urban greenery of Lublin and their aphidofauna. Aphids and other Hemipterous Insects, 16: 37-47.

Chmura D., Adamski P., Denisiuk Z., 2013 - How do plant communities and flower visitor relate? A case study of semi-natural xerothermic grasslands. - Acta Soc. Bot. Pol., 82(2): 99-105.

CichockA E., LUBIARz M., 2010 - Landscape type and species richness and composition of Arthropoda. Part I. Agricultural landscape. - Aphids and Other Hemipterous Insects, 16: 59-78.

Cremene C., Groza G., Rakosy L., Schileyko A.A., Baur A., ERHARDT A., BAUR B., 2005 - Alterations of steppelike grasslands in Eastern Europe: a threat to regional biodiversity hotspots. - Conserv Biol., 19(5): 1606-1618.

Czylok A., 1983 - Zgrupowania mszyc (Homoptera, Aphidodea) wybranych zbiorowisk leśnych okolic Pińczowa. - Acta Biol., 13: 114-130. (in Polish)

Czylok A., Gorczyca J., Halaj R., Klimaszewski S.M., Wojciechowski W., 1991 - Fauna mszyc (Homoptera, Aphidodea) zwałowisk odpadów węglowych i cynkowych 
województwa katowickiego. - Acta Biol. Siles., 18: 108117. (in Polish)

Czylok A., Halaj R., Woźnica A., 1988 - Mszyce (Homoptera, Aphidomorpha) zbiorowisk roślinnych Bieszczadów Zachodnich. - Acta Biol. Siles., 10: 93-109. (in Polish)

CZYlok A., WoJcIechowski W., 1987 - Communities of aphids (Homoptera, Aphidoidea) of the Xerothermic Vegetation in Niecka Nidziańska. - Acta Biol. Siles., 6: 37-41.

Dengler J., Oldeland J., Jansen F., Chytrý M., Ewald J., Finckh M., Glöckler F., Lopez-GonZalez G., Peet R.K., SCHAMINÉE J.H.J., 2012 - Vegetation databases for the 21st century. - Biodiversity \& Ecology, 4: 325-325.

DePa Ł., WojciechowsKi W., 2009 - Aphids (Hemiptera: Aphidinea) of Garb Tarnogórski and their trophobiotic relations with ants. - Annals of the Upper Silesian Museum (Entomology), 18: 5-106.

DEPA Ł., MrÓz E., 2012 - Uroleucon leontodontis (Hille Ris Lambers, 1939) - an aphid species new to Poland with the description of its hitherto unknown alate morph (Hemiptera: Aphididae). - Genus, 23: 223-227.

DoBRZAŃSKA J., 1955 - Flora and ecological studies on calamine flora in the district of Bolestaw and Olkusz. Acta Soc. Bot. Pol., 24: 357-408.

DuraK R., DuraK T., BorowiaK-SobKowiak B., 2011 Charakterystyka mszyc (Hemiptera: Aphidoidea) z zastosowaniem funkcjonalnych grup ekologicznych na przykładzie lasów bukowych w Beskidach Wschodnich. Wiadomości entomologiczne, 30: 7-16. (in Polish)

DURAK R., WojcIECHOWSKI W., 2005 - Aphid (Hemiptera: Aphidoidea) communities in different forest associations (Vaccinio-Piceetes and Querco-Fagetea classes) of the Kolbuszowa Plateau. - Aphids and Other Hemipterous Insects, 11: 39-52.

EASTOP V.F., 1995 - Aphids, plants and other organisms. Korean J. Appl. Ent., 34: 1-8.

European Commission DG Environment, 2013 Calaminarian grasslands of the Violetalia calaminariae. Interpretation Manual of European Union Habitats. $<$ http://eunis.eea.europa.eu/habitats/10113> accessed $1^{\text {st }}$ January 2016.

Everitt B.S., Landau S., Leese M., Stahl D., 2011 Cluster analysis. John Wiley \& Sons, Ltd., Chichester, $346 \mathrm{pp}$.

GoRCZYCA J., 1994 - Mirid communities (Heteroptera: Miridae) of the plant assemblages in Wyzyna Czestochowska. - Annals of the Upper Silesian Museum in Bytom. Natural History, 14: 33-68.

GRESZTA J., MoraWSKI S., 1972 - Rekultywacja nieużytków poprzemystowych. PWRiL, Warszawa, 263 pp. (in Polish)

GrodziŃSKA K., SZAREK-ŁUKASZEWSKA G., 2009 - Heavy metal vegetation in the Olkusz region (Southern Poland) - preliminary studies. - Pol. Bot. J., 54: 105-112.

HaILA Y., 2002 - A conceptual genealogy of fragmentation research: from island biogeography to landscape ecology. - Ecol. Appl., 12: 321-334.

HaŁAJ R., 1993 - The fauna of aphids (Homoptera, Aphidinea) of the selected high peat-bogs in the Polish Carpathians. - Acta Biol. Siles., 22: 92-104.

HaŁAJ R., 1999 - Nowe gatunki mszyc (Homoptera: Aphidinea) dla Wyżyny Śląskiej. - Acta Ent. Siles., 5-6: 51. (in Polish)

HAŁAJ R., OsIADACZ B., 2014 - Ctenocallis israelica - first record in Central Europe, with a note on the genus Ctenocallis. - Turk. J. Zool., 38: 35-41.

Hataj R., Osiadacz B., 2016 - Hormaphididae,
Mindaridae, Phloeomyzidae, Thelaxidae. - Keys for the Identification of Polish Insects XVII(5c) [in press].

Halas R., WoJciechowski W., 1996 - Zgrupowania mszyc (Homoptera, Aphidinea) związane ze zbiorowiskami murawowymi z klas Festuco-Brometea i SedoScleranthetea Wyżyny Częstochowskiej. - Acta Biol. Siles., 29: 83-105. (in Polish)

HAŁAJ R., WoJcIEchowsKi W., 1997 - Zgrupowania mszyc (Homoptera, Aphidinea) zwiazane z niektórymi zbiorowiskami z klas Vaccinio-Picetea, Alnetea glutinosae $i$ Querco-Fagetea okolic Olsztyna k. Częstochowy. - Acta Biol. Siles., 30: 61-83. (in Polish)

HANSKI I., OvaSKAINEN O., 2003 - Metapopulation theory for fragmented landscapes. - Theor. Popul. Biol., 64: 119127.

HeIE O.E., 1980 - The Aphidoidea of Fennoscandia and Denmark I. Mindaridae, Hormaphidae, Thelaxidae, Anoecidae and Pemphigidae. - Fauna Ent. Scand., 9: 1236.

HEIE O.E., 1986 - The Aphidoidea of Fennoscandia and Denmark III. Pterocommatinae and Aphidinae, Aphidini. - Fauna Ent. Scand., 17: 1-314.

Holman J., 2009 - Host Plant Catalog of Aphids, Palaearctic Region. Springer, Dordrecht, 1140 pp.

JĘDRZEJCZYK-KORYCIŃSKA M., 2011 - Tajemnicze życie pośród hałd. - Problemy środowiska i jego ochrony, 19: 114-130. (in Polish)

Kapusta P., Szarek-Łukaszewska G., Rodzińska K., GoDzIK B., 2010 - Murawy galmanowe okolic Olkusza (poludniowa Polska) i problemy ich ochrony. - Chrońmy Przyrodę Ojczystą, 66: 27-34. (in Polish)

Kirchner S.M., Hiltunen L., Döring T.F., Virtanen E., Palohuhta J.P., Valkonen J.P.T., 2013 - Seasonal Phenology and Species Composition of the Aphid Fauna in a Northern Crop Production Area. - PLoS ONE, 8: e71030.

Kowolik M., Szarek-Łukaszewska G., JęDrzejczyKKORYCIŃSKA M., 2010 - Użytek ekologiczny „Pleszczotka górska" w cynkowo-ołowiowym terenie pogórniczym potrzeba aktywnej ochrony. - Chrońmy Przyrodę Ojczystą, 66: 35-38. (in Polish)

Lin Y-P., Cook D.H., Gullan P.J., CoOK L.G. 2015 - Does host-plant diversity explain species richness in insects? A test using Coccidae (Hemiptera). - Ecol. Entomol., 40: 299-306.

Losos J.B., RickLefs R.E., 2009 - The Theory of Island Biogeography Revisited. Princeton University Press, Princeton, New Jersey, 496 pp.

MAŃCZYK A., RosTAŃSKI A., 2003 - Flora naczyniowa wybranych zwałów pocynkowych miasta Ruda Sląska. Archiwum Ochrony Środowiska, 29: 107-113. (in Polish)

Matuszkiewicz W., 2012 - Przewodnik do oznaczania zbiorowisk roślinnych Polski. PWN, Warszawa, 540 pp.

Nieto Nafría J.M., Mier Durante M.P., Garcio Prieto F., Pérez Hidalgo N., 2005 - Hemiptera, Aphididae III. Fauna Ibérica, 28: 1-362.

NowaK T., JęDrZEJCZYK-KorycińsKa M., Kapusta P., SZAREK-ŁUKASZEWSKa G., 2015 - Characteristics of the vascular plant flora in the Olkusz Ore-bearing Region. In: Natural and historical values of the Olkusz Ore-bearing Region, Godzik B. Ed., W. Szafer Institute of Botany, Polish Academy of Sciences, Krakow, pp.147-171.

Nowak T., Kapusta P., JędrzejczyK-Korycińska M., Szarek-ŁukASZEWSKa G., GodzIK B., 2011 - The vascular plants of the Olkusz Ore-bearing Region. W. Szafer Institute of Botany, Polish Academy of Sciences, Krakow, 226 pp. 
OsIADACZ B., HataJ R., 2009 - The Aphids (Hemiptera: Sternorrhyncha: Aphidinea) of Poland. A Distributional Checklist. - Pol. Entomol. Monogr., 6: 1-96.

Osiadacz B., HaŁAJ R., 2011 - Biological remarks on Uroleucon (Uromelan) rapunculoidis (Börner, 1939) (Hemiptera: Aphidoidea) with a new host plant record Campanula cervicaria $L$. - Wiadomości entomolologiczne, 30: 197-204.

Osiadacz B., Hataj R., 2015 - Aphids in jeopardy? Aphid communities on xerothermic habitats. - Biologia, 70(8): 1118-1135.

OSIADACZ B., WIECZOREK K., 2003 - Mszyce (Hemiptera: Aphidoidea) wybranych parków Bytomia. - Acta Ent. Siles., 11: 39-46. (in Polish)

Osiadacz B., WieczoreK K., 2007 - Aphids (Hemiptera, Aphidoidea) on selected marshy communities. - Aphids and Other Hemipterous Insects, 13: 75-81.

Osiadacz B., Wojciechowski W., 2008 - Aphids (Hemiptera: Aphidinea) of the Ojców National Park. Structure and origin of fauna. The Monograph. - Annals of the Upper Silesian Museum in Bytom. Natural History, 18: 1-172.

ÖCKINGER E., ERIKSSON A.K., SMITH H.G., 2006 - Effects of grassland abandonment, restoration and management on butterflies and vascular plants. - Biol Conserv., 133(3): 291-300.

Quednau F.W., 2003 - Atlas of the drepanosiphine aphids of the world. Part II. Panaphidini Oestlund, 1923 Panaphidina Oestlund 1923 (Hemiptera: Aphididae: Calaphidinae). - Memoirs of the American Entomological Institute, 72: 1-301.

R CORE TEAM, 2015 - R: A Language and Environment for Statistical Computing. R Foundation for Statistical Computing, Vienna.

ROSTAŃSKI A., 1997a - Flora spontaniczna hałd Górnego Śląka. - Archiwum Ochrony Środowiska, 23: 159-165. (in Polish)

RosTAŃSKi A., 1997b - Rośliny naczyniowe terenów o wysokim stopniu skażenia metalami ciężkimi. - Acta Biol. Siles., 30: 56-85. (in Polish)

RUSZKOWSKA M., WILKANIEC B., 2002 - Urban fauna of aphids (Homoptera, Aphidoidea) related to trees and shrubs in the Poznan district. - Journal Plant Protection Research, 42: 205-214.

SKUBAŁA K., 2011 - Vascular flora of sites contaminated with heavy metals on the example of two post-industrial spoil heaps connected with manufacturing of zinc and lead products in Upper Silesia. - Arch. Environ. Prot., 37: 57-74.

SzareK-Łukaszewska G., Grodzińska K., 2011 Grasslands of a $\mathrm{Zn}$-Pb post-mining area (Olkusz Orebearing Region, S Poland). - Pol. Bot. J., 56: 245-260.

SzElEGIEwICZ H., 1974 - Mszyce (Homoptera, Aphidodea) Mierzei Wiślanej ze szczególnym uwzględnieniem wydm nadmorskich. - Fragmenta Faunistica, 19: 349-393. (in Polish)

Szelegiewicz H., 1976 - Materiaty do fauny mszyc (Homoptera, Aphidodea) Pobrzeża Battyku. - Fragmenta Faunistica, 20: 369-406. (in Polish)

Szwedo J., GęBicki C., WegiereK P., 1998 - Leafhopper communities (Homoptera, Auchenorrhyncha) of selected peat-bogs in Poland. - Annals of the Upper Silesia Museum in Bytom, Natural History, 15: 154-176.

WIERZBICKA M., RostAŃSKi A., 2002 - Microevolutionary changes in the ecotypes of calamine waste heap vegetation near Olkusz, Poland: a review. - Acta Biol. Cracov. Bot., 44: 7-19.

WiLKANIEC B., LEWANDOWSKI R., BOROWIAK-SOBKOWIAK B., 2012 - The effectiveness of catching aphids (Hemiptera: Sternorrhyncha: Aphidinea) in moericke and light traps. - Journal of Plant Protection Research, 52: 259-263.

Wilkaniec B., Piekarska-Boniecka H., Suder A., 1999 Aphids (Homoptera, Aphidodea) of refuge habitats in the agricultural landscape of Wielkopolska. - Aphids and Other Hemipterous Insects, 7: 117-123.

Wojciechowski W., Halaj R., Kolodziej B., 1989 Mszyce (Homoptera, Aphidomorpha) Pustyni Błędowskiej. - Acta Biol. Siles., 13: 31-43. (in Polish)

Wojciechowski W., WoźnicA A., 1989 - Mszyce (Homoptera, Aphidomorpha) Sudetów Środkowych. Acta Biol. Siles., 13: 43-62. (in Polish) 
